PUPT-2036

\title{
Wilson Loops, D-BRANEs, AND Reparametrization PATH-INTEGRALS
}

\author{
Vyacheslav S. Rychkov" \\ Department of Mathematics \\ $\&$ \\ Joseph Henry Laboratories \\ Princeton University \\ Princeton, NJ 08540
}

\begin{abstract}
We study path-integrals over reparametrizations of the world-sheet boundary. Such integrals arise when string propagates between fixed space-time contours. In gauge/string duality they are needed to describe gauge theory Wilson loops. We show that (1) in AdS/CFT, the integral is well defined and gives a finite 1-loop correction to the Wilson loop; (2) in critical string theory, the integral is UV divergent, and fixed contour amplitudes are off shell. In the second case, we show that the divergences can be removed by renormalizing the contour. We calculate the 2-loop contour $\beta$-function and explain how it is related to the D0-brane effective action. We also apply this method to compute the first $\alpha^{\prime}$ correction to the effective action of higher dimensional branes.
\end{abstract}

April 2002

${ }^{1}$ rytchkov@math.princeton.edu 


\section{Introduction}

Gauge fields are believed to have a dual description in terms of strings, representing their flux lines. This belief has been considerably strengthened in recent years, although a systematic theory is still missing. The most important problem is to move beyond the supergravity approximation on string theory side of the duality. Sooner or later it has to be done, if our final goal is to describe physically interesting asymptotically free theories.

The gauge theory Wilson loop $W[C]$ has played an important role in discussions of gauge/string duality, both in the early period [1, 2] and in the recent work [3, 国. In the dual picture it has a very suggestive description as a path-integral over string world-sheets with a fixed boundary contour $C$. In the supergravity limit, this integral is dominated by the corresponding minimal surface. This paper grew out of attempts to understand quantum corrections to this supergravity result.

A starting point of our discussion is an old observation [5, [6] that an amplitude describing string propagating between fixed space-time contours reduces in conformal gauge to a path-integral over boundary reparametrizations. We apply this idea in the analysis of 1-loop corrections to the AdS/CFT ansatz for the Wilson loop [0, ㄱ‥ Our main result is that for contours $C$ lying strictly on the boundary of the AdS space, the 1-loop correction is finite, and the world-sheet conformal invariance is preserved. On the contrary, for contours lying inside AdS the reparametrization path-integral is logarithmically divergent, and the corresponding amplitude is off shell and ill defined.

Fixed contour amplitudes in flat space are off shell just as the amplitudes for contours inside AdS. However, we decided to study the corresponding reparametrization pathintegral in great detail. One reason for this study is to gain practical knowledge about reparametrization path-integrals in general. The flat space background provides a good model example, because the action in this case is known explicitly, and a rather precise analysis can be carried out.

A second reason comes from an observation that the $\sigma$-model description of D0-branes gives rise to a formally equivalent path-integral. This powerful analogy lead us to conjecture that the corresponding non-local field theory of reparametrizations is renormalizable, in the sense that all the divergences can be removed by adding counterterms changing the shape of the contour. We checked this conjecture to the 2-loop order in the Feynman diagram expansion, and found in particular that the divergences cancel provided the D0-brane equations of motion are satisfied.

There exists a natural generalization of the above result to the case of higher dimensional branes, in which the role of reparametrizations is played by maps from $S^{1}$ into the brane world-volume. We use this fact to find the first $\alpha^{\prime}$ correction to the $\mathrm{D} p$-brane low energy effective action, and show that the result agrees with the action computed from the $S$-matrix amplitudes. 
The exact structure of the paper is as follows. In Section 2 we review the general modern picture of gauge/string duality, discuss the string theory ansatz for the Wilson loop, and explain the appearance of reparametrization path-integrals. We show that the 1-loop correction is divergent for contours in flat space, and finite for contours on the boundary of AdS. We also make a foray into the subject of loop equations, reviewing old and recent work and discussing possibilities for future research.

In Section 3 we study renormalization of the flat space reparametrization path-integrals. We calculate the 2-loop contour $\beta$-function, and explain how it is related to the D0-brane effective action. Before treating the general case, we also consider the circular contour example.

In Section 4 we deal with the $\mathrm{D} p$-brane case. We compute a 2-loop condition for cancellation of logarithmic divergences in a corresponding path-integral, and interpret this condition as an equation of motion following from a low energy effective action.

Section 5 is a short conclusion. Appendices A and B are devoted to the details of Feynman diagram analysis.

\section{Wilson loop in gauge/string duality}

\subsection{General picture}

The goal of the gauge/string duality program is to find a string Lagrangian for $4 \mathrm{~d}$ gauge theory color-electric flux lines. This Lagrangian must give permanent confinement at large distances, and at the same time reproduce high-energy asymptotic freedom predictions.

It is well understood by now [9, , 4, 10] that for the pure Yang-Mills theory such "confining strings" must propagate in a 5d gravitational background of the form

$$
d s^{2}=A(y)\left(d y^{2}+d x_{\mu}^{2}\right), \quad A(y) \sim y^{-2} \quad(y \rightarrow 0) .
$$

The gauge theory itself lives at the "absolute" $y=0$ of this space.

The background (2.1) will contain extra dimensions if the $4 \mathrm{~d}$ gauge theory in question has some extra matter fields, as it happens for the much studied AdS/CFT example of the Yang-Mills theory with $\mathcal{N}=4$ supersymmetry (see [1] for a review). In this case conformal symmetry fixes

$$
A(y)=\frac{R^{2}}{y^{2}},
$$

so that the metric (2.1) describes the $A d S_{5}$ space, while the full background is $A d S_{5} \times S^{5}$ due to the 6 scalars present in the supersymmetric Yang-Mills. 
In physically interesting non-conformal cases the factor $A(y)$ will have logarithmic corrections

$$
A(y) \sim \frac{R^{2}}{y^{2}}\left[\log \left(\frac{r_{*}}{y}\right)\right]^{\alpha}
$$

near $y=0$, corresponding to the gauge theory logarithmic corrections to the Coulomb law at short distances. Moreover, the space (2.1) will terminate at some finite $y$, leading to confinement. These features have also been observed for gravity duals of strongly coupled gauge theories with logarithmic RG flows [12].

Eventually, gauge/string duality must provide precise identification of gauge invariant operators of the gauge theory with vertex operators of string theory on the background (2.1), so that the corresponding field theory correlators and string theory scattering amplitudes are equal. In the standard AdS/CFT correspondence [11] the gauge theory coupling is strong, while the background (2.1) is weakly curved, and the supergravity approximation to the full string theory is applicable.

For weakly coupled or asymptotically free gauge theories we cannot use supergravity and must solve the string $\sigma$-model with the world sheet action

$$
S=\frac{1}{4 \pi \alpha^{\prime}} \int d^{2} \xi \sqrt{g} g^{a b} \partial_{a} X^{M} \partial_{b} X^{N} G_{M N}(X)+\ldots
$$

where $G_{M N}(X)$ is metric (2.1) with $X=\left(y, x_{\mu}\right)$, and ... denotes terms in the action corresponding to extra dimensions, world-sheet fermions, and RR backgrounds needed to stabilize the space (2.1). In conformal cases there are explicit proposals for the full world-sheet action [10, 13]. These $\sigma$-models have not been solved so far.

\subsection{Wilson loop}

The gauge theory Wilson loop operators

$$
W[C]=\frac{1}{N}\left\langle\operatorname{Tr} \operatorname{Pexp} \oint_{C} A_{\mu} d x_{\mu}\right\rangle_{\mathrm{YM}}
$$

plays a special role in the gauge/string correspondence. In the dual picture it should be described by an open string amplitude with the string boundary tracing the contour $C$ [1]. In other words, the Wilson loop must be given by a path-integral

$$
W\left[c_{\mu}(\sigma)\right]=\int[\mathcal{D} X \mathcal{D} g \ldots] \exp -S[X, g \ldots]
$$

with the boundary conditions

$$
x_{\mu}(\sigma, 0)=c_{\mu}(\sigma), \quad y(\sigma, 0)=0 .
$$


At present no general methods to work with (2.6) are known. For conformal theories (2.2) it is possible to consider the strong coupling limit

$$
\sqrt{\lambda}=\frac{R^{2}}{4 \pi \alpha^{\prime}} \gg 1
$$

In this case the integral (2.6) can be computed in the classical approximation as [0, 8]

$$
W\left[c_{\mu}(\sigma)\right] \approx \exp -\sqrt{\lambda} A_{\min }
$$

where $A_{\text {min }}$ is the area of the minimal surface in $A d S_{5}$ bounded by the contour $c_{\mu}(\sigma)$. World-sheet fermions and RR fields do not contribute in this approximation.

As a first step towards a full theory, one can try to understand corrections to (2.9) perturbatively, as an expansion in $\lambda^{-1}$.

\subsection{Loop equation}

It has been known for a long time [14, 2, 5, 15] that gauge theory Schwinger-Dyson equations in the large $N$ limit give a closed equation for the Wilson loop. This "loop equation" has the form

$$
\widehat{L}(\sigma) W[C]=W\left[C_{1}\right] * W\left[C_{2}\right],
$$

where the "loop Laplacian"

$$
\widehat{L}(\sigma)=\lim _{\varepsilon \rightarrow 0} \int_{\sigma-\varepsilon}^{\sigma+\varepsilon} d \sigma^{\prime} \frac{\delta^{2}}{\delta c_{\mu}(\sigma) \delta c_{\mu}\left(\sigma^{\prime}\right)}
$$

is the operator which picks up the $\delta$-function singular term in the second variational derivative. The r.h.s.

$$
W\left[C_{1}\right] * W\left[C_{2}\right]=-\lambda \oint_{C} \delta^{(4)}(y-c(\sigma)) d y_{\mu} \dot{c}_{\mu}(\sigma) W\left[C_{1}\right] W\left[C_{2}\right]
$$

is nonzero only for $\sigma$ corresponding to a point of self-intersection, in which case it gets contribution from the "halves" $C_{1}$ and $C_{2}$ obtained from $C$ by splitting at that point. It is identically zero for non-selfintersecting contours.

Strictly speaking, Eq. (2.10) is derived in a regularized theory. We would like to promote it to a well-defined equation for the finite, renormalized Wilson loop. So far it has not been done, mainly because it is unclear how to handle self-intersecting loops. If $C$ is a smooth non-selfintersecting contour, then $W[C]$ has a finite renormalized value [16]. 
However, self-intersecting Wilson loops contain additional logarithmic divergences, and so do the loops with corner points into which self-intersecting loops split [2, 17].

To avoid these difficulties, we may decide to limit ourselves with non-selfintersecting loops. In this case it was shown in [18] that the renormalized loop equation

$$
\widehat{L}(\sigma) W[C]=0
$$

is well defined.

Eq. (2.13) by itself would not be sufficient to nonambiguosly recover the Wilson loop functional. For example, it does not distinguish between abelian and non-abelian gauge theories. Perhaps it may be supplemented by some sort of "boundary conditions" for nearly self-intersecting loops, which will make the identification unique. At present this issue is not settled. However, even in the form (2.13) the loop equation may be used to test the gauge/string duality ansatz (2.6).

For strongly coupled conformal gauge theory this test was performed in [18, 19], where we showed that the classical approximation (2.9) satisfies (2.13) for any contour $C$ precisely in the critical dimension $D=4$. This conclusion was reached by studying the second variational derivative of the nonlinear functional $A_{\min }[C]$ in the short-distance limit $\sigma^{\prime} \rightarrow \sigma$. We found the singular momentum behavior of the form

$$
\begin{aligned}
& \int \frac{\delta^{2} A_{\min }}{\delta c_{\mu}\left(\sigma-\frac{h}{2}\right) \delta c_{\mu}\left(\sigma+\frac{h}{2}\right)} e^{i p h} d h \\
= & (1-D)|p|^{3}+(D-4) \ddot{c}^{2}(\sigma)|p|+(4-D) \ddot{c}_{\mu}(\sigma) \frac{\delta A_{\min }}{\delta c_{\mu}(\sigma)} p^{0}+\ldots,
\end{aligned}
$$

where ... denotes terms which are $O\left(p^{-1}\right)$ for $p \rightarrow \infty$. The functional $A_{\min }$ is invariant under reparametrizations $c_{\mu}(\sigma) \rightarrow c_{\mu}(f(\sigma))$, and formula (2.14) is written in the natural gauge $\dot{c}^{2} \equiv 1$.

In the coordinate space the first two terms in the r.h.s. of (2.14) produce non-local singularities proportional to $h^{-4}$ and $h^{-2}$, while the third term corresponds to the local $\delta(h)$ singularity. The loop Laplacian $\widehat{L}(s) W[C]$ is thus proportional to the coefficient in front of the third term, and we see that indeed (2.13) is satisfied provided that $D=4$.

\subsection{Quantum corrections}

As we move to smaller $\lambda$, quantum corrections to the classical formula (2.9) must become important . At present the structure of these corrections and their dependence on the contour are not understood. In particular, I do not know if the loop equation

\footnotetext{
${ }^{2}$ Although for the Wilson loop in $\mathcal{N}=4 \mathrm{SYM}$ formula $(2.9)$ is conjectured to hold to all orders in $\lambda$ if $C$ is a circle 20, 21].
} 
(2.13) continues to hold when the corrections are taken into account. If it turns out that it does not, this will perhaps have an interpretation based on the unavoidable presence of matter fields in conformal gauge theories [18].

One-loop corrections to (2.9) for type IIB string on $A d S_{5} \times S^{5}$ described by the GreenSchwarz action were studied in an important paper 22] (see also 23]). As it was shown in [22], logarithmic divergences in bosonic and fermionic determinants coming from worldsheet oscillations cancel. This important conclusion (related to the fact that $A d S_{5} \times S^{5}$ is an exact closed string background) leaves however out the following puzzle.

We do expect of course that the first quantum correction to (2.9) be finite, and conformal invariance in (2.6) be preserved on the 1-loop level. However, conformal invariance would be broken had we put contour $C$ at $y=\varepsilon>0$ rather than on the absolute.

Consider for instance standard critical string theory in flat space. It is well known that amplitudes with fixed boundary would be off shell, i.e. not conformally invariant, except for a very special class of contours (straight lines, also known as D0-branes).

Path-integral (2.6) with a contour $C$ inside AdS must behave similarly to the flat space integral in this respect. Only when the contour is pushed all the way to the absolute, where the metric is singular, can we expect restoration of conformal invariance. Apparently we are missing something, because the cancellation of bosonic and fermionic divergences observed in [22] does not seem to depend on the precise position of the contour.

To resolve the puzzle, it is necessary to realize that besides the world-sheet determinants, stringy Wilson loop (2.6) receives a 1-loop correction from oscillations of the classical action induced by reparametrizations of the boundary contour. This is where reparametrization path-integrals come into play. This correction must be analyzed separately to decide if the conformal invariance is preserved. In particular, it is the structure of this correction that creates the difference between contours on the boundary of AdS on the one hand, and contours in flat space and inside AdS on the other. Below we explain in detail how this happens, first in flat space, and then in AdS.

\subsection{Flat space example}

Let us first recall what goes wrong with fixed boundary amplitudes in flat space, considering bosonic string for simplicity. We are to consider the path-integral

$$
Z=\int\left[\mathcal{D} X^{\mu} \mathcal{D} g\right] \exp \left(-\frac{1}{4 \pi \alpha^{\prime}} S_{P}\right), \quad S_{P}=\int d \sigma d \tau \sqrt{g} g^{a b} \partial_{a} X^{\mu} \partial_{b} X^{\mu}
$$

with the boundary conditions

$$
X^{\mu}(\sigma, 0)=c^{\mu}(\sigma)
$$

where $c^{\mu}(\sigma)$ is a contour in flat $D$-dimensional space. The open string world-sheet with disk topology is parametrized by the upper half-plane. 
In conformal gauge the action becomes

$$
S=\int d \sigma d \tau(\partial X)^{2}
$$

World-sheet oscillations and ghosts give rise to determinants that cancel each other provided that $D=26$. The answer we seem to be getting is

$$
Z \stackrel{?}{=} \exp \left(-\frac{1}{4 \pi \alpha^{\prime}} S_{\mathrm{cl}}\left[c_{\mu}(\sigma)\right]\right)
$$

where $S_{\mathrm{cl}}$ is the classical action for the solution of the Dirichlet problem on the upper half-plane with the given boundary conditions

$$
S_{\mathrm{cl}}=\int \frac{d p}{2 \pi}|p| c^{\mu}(p) c^{\mu}(-p)
$$

However, Eq. (2.18) is not, cannot be correct. This can already be seen from the fact that in the classical $\alpha^{\prime} \rightarrow 0$ limit (2.15) must be dominated by the surface with minimal area rather than minimal energy. Even more, (2.15) is formally invariant under reparametrizations $\sigma \rightarrow \alpha(\sigma)$, while the classical Dirichlet action $S_{\mathrm{cl}}$ is not.

The correct answer is

$$
Z=\int[\mathcal{D} \alpha(\sigma)] \exp \left(-\frac{1}{4 \pi \alpha^{\prime}} S_{\mathrm{cl}}\left[c_{\mu}(\alpha(\sigma))\right]\right)
$$

where the path-integral is over the group of boundary reparametrization. This expression is manifestly reparametrization invariant (modulo possible quantum anomalies). It also possesses the right classical limit, because

$$
\min _{\{\alpha(\sigma)\}} S_{\mathrm{cl}}\left[c_{\mu}(\alpha(\sigma))\right]=\text { minimal area } .
$$

The reason why $(2.20)$ rather than $(2.18)$ follows from (2.15) is that fixing conformal gauge on the disc is in general impossible unless we allow diffeomorphisms reparametrizing the boundary. After we trade the integral over metrics for an integral over Diff $\times W e y l$, the part $\operatorname{Diff} f_{0} \times W e y l$ produces but an infinite volume factor, while the $\operatorname{Diff}\left(S^{1}\right)$ component gives rise to (2.20). More details can be found in Appendix A.

Eq. (2.20) has appeared in the literature before, see e.g. [5, 6], but the dynamical consequences of this representation to the best of my knowledge have not been explored.

Since we expect that for a general contour conformal invariance in (2.15) is broken, the integral (2.20) must contain logarithmic divergences, and it indeed does. Let us see 
how this happens in the 1-loop approximation. Without loss of generality, we may assume that $\alpha_{*}(\sigma) \equiv \sigma$ provides minimum in (2.21) $)^{\text {. Th }}$ The action in coordinate space becomes

$$
S_{\mathrm{cl}}\left[c_{\mu}(\sigma+\beta(\sigma))\right]=-\frac{1}{\pi} \int d \sigma d \sigma^{\prime} \frac{c_{\mu}(\sigma+\beta(\sigma)) c_{\mu}\left(\sigma^{\prime}+\beta\left(\sigma^{\prime}\right)\right)}{\left(\sigma-\sigma^{\prime}\right)^{2}}
$$

The part of the action quadratic in $\beta(\sigma)$ is given by

$$
S_{\mathrm{II}}=-\frac{1}{\pi} \int d \sigma d \sigma^{\prime} \frac{\dot{c}_{\mu}(\sigma) \dot{c}_{\mu}\left(\sigma^{\prime}\right)}{\left(\sigma-\sigma^{\prime}\right)^{2}} \beta(\sigma) \beta\left(\sigma^{\prime}\right)+\frac{1}{2} \int d \sigma \frac{\delta S_{\mathrm{cl}}}{\delta c_{\mu}(\sigma)} \ddot{c}_{\mu}(\sigma) \beta^{2}(\sigma)
$$

where

$$
\frac{\delta S_{\mathrm{cl}}}{\delta c_{\mu}(\sigma)}=-\frac{2}{\pi} \int d \sigma^{\prime} \frac{c_{\mu}\left(\sigma^{\prime}\right)}{\left(\sigma-\sigma^{\prime}\right)^{2}} .
$$

For later reference notice that we have

$$
\frac{\delta S_{\mathrm{cl}}}{\delta \beta(\sigma)}=\frac{\delta S_{\mathrm{cl}}}{\delta c_{\mu}(\sigma)} \dot{c}_{\mu}(\sigma) \equiv 0
$$

since the linear in $\beta(\sigma)$ term in the action has to vanish.

The first quantum correction in (2.20) corresponds to the path-integral

$$
\int[\mathcal{D} \beta] \exp \left(-S_{\mathrm{II}}\right)
$$

This integral contains a logarithmic divergence. Indeed, from the first term in $S_{\text {II }}$ we see that the propagator of the field $\beta(\sigma)$ in the mixed representation behaves like

$$
\langle\beta(p) \beta(-p)\rangle=\frac{1}{2|p| \dot{c}^{2}(\sigma)}+O\left(p^{-2}\right) \quad(p \rightarrow \infty)
$$

It follows that

$$
\left\langle\beta^{2}(\sigma)\right\rangle=\frac{\log \Lambda}{2 \pi \dot{c}^{2}(\sigma)}+\text { finite },
$$

where $\Lambda$ is the momentum cutoff.

Since the second term in $S_{\text {II }}$ involves $\beta^{2}(\sigma)$, we get a logarithmically divergent contribution to the effective action $F=-\log Z$

$$
F_{\mathrm{div}}=\frac{\log \Lambda}{4 \pi} \int d \sigma \frac{\delta S_{\mathrm{cl}}}{\delta c_{\mu}} \frac{\ddot{c}_{\mu}}{\dot{c}^{2}}
$$

\footnotetext{
${ }^{3}$ Otherwise we have to reparametrize $c_{\mu}(\sigma) \rightarrow c_{\mu}\left(\alpha_{*}(\sigma)\right)$.
} 
The same divergence can be derived in a different way, by using heat-kernel methods to analyze the determinant of the integral operator corresponding to $S_{\mathrm{II}}$.

It is worth noting that the divergence $(2.29)$ is non-local and cannot be removed by adding local counterterms to the contour action. Conformal invariance would be indeed broken unless it canceled. The most general local condition for this to happen is

$$
\ddot{c}(\sigma) \equiv 0
$$

This means that the contour has to be a straight line for (2.20) to be well defined. The straight line boundary condition would be identical to having a D0-brane, if it were not for a subtle difference [3]. Namely, in (2.20) the surface is attached to the contour without folds, while for the D0-branes the folds are allowed.

I will have a lot more to say about the path-integral (2.20) and its relation to the bosonic D-branes in Sections 3 and 4 of this paper. Now let us return to the Wilson loop in AdS.

\subsection{Wilson loop in $A d S$ is 1-loop finite}

In the AdS case, a 1-loop analysis very similar to the one given in the previous section can be carried out. As I mentioned in Section 2.4, world-sheet oscillations do not lead to logarithmic divergences in this order. As a result, all possible troubles are connected with the AdS analogue of $(2.20)$ 目

$$
\int[\mathcal{D} \alpha(\sigma)] \exp \left(-\frac{1}{4 \pi \alpha^{\prime}} S_{A d S}\left[c_{\mu}(\alpha(\sigma))\right]\right) .
$$

Here $S_{A d S}\left[c_{\mu}(\sigma)\right]$ is the classical Dirichlet action in the AdS space

$$
S_{A d S}=\min \int d^{2} \xi \frac{\left(\partial x_{\mu}\right)^{2}+(\partial y)^{2}}{y^{2}}
$$

with boundary conditions (2.16).

Unlike in flat space, no explicit formula similar to (2.19) exists for $S_{A d S}$. However, to find the 1-loop divergences, it is sufficient to understand short-distance singularities of the quadratic action. In other words, we have to study

$$
\frac{\delta^{2} S_{A d S}}{\delta \beta(\sigma) \delta \beta\left(\sigma^{\prime}\right)}=\frac{\delta^{2} S_{A d S}}{\delta c_{\mu}(\sigma) \delta c_{\nu}\left(\sigma^{\prime}\right)} \dot{c}_{\mu}(\sigma) \dot{c}_{\nu}\left(\sigma^{\prime}\right)+\frac{\delta S_{A d S}}{\delta c_{\mu}(\sigma)} \ddot{c}_{\mu}(\sigma) \delta\left(\sigma-\sigma^{\prime}\right) .
$$

\footnotetext{
${ }^{4}$ This integral over reparametrizations was disregarded in [22].
} 
The latter formula folows by Taylor expanding $c_{\mu}(\sigma+\beta(\sigma))$.

Short-distance limit of the second derivative of $S_{A d S}$ was analyzed in [19], where we found that

$$
\int \frac{\delta^{2} S_{A d S}}{\delta c_{\mu}\left(\sigma-\frac{h}{2}\right) \delta c_{\mu}\left(\sigma+\frac{h}{2}\right)} e^{i p h} d h=\left(3 \frac{\dot{c}_{\mu} \dot{c}_{\nu}}{\dot{c}^{4}}-\frac{\delta_{\mu \nu}}{\dot{c}^{2}}\right)|p|^{3}+\mathrm{const} \frac{\dot{c}_{[\mu} \ddot{c}_{\nu]}}{\dot{c}^{4}} p^{2}+O(p)
$$

All coefficients in the r.h.s. are evaluated at $\sigma$. It is crucial for what I am going to say that the $p^{2}$ term is multipled by an antisymmetric tensor (in fact, this follows from $\sigma \rightarrow-\sigma$ symmetry of the problem). After substituting into (2.33), the $p^{2}$ term vanishes, and we obtain

$$
\int \frac{\delta^{2} S_{A d S}}{\delta \beta\left(\sigma-\frac{h}{2}\right) \delta \beta\left(\sigma+\frac{h}{2}\right)} e^{i p h} d h=2|p|^{3}+O(p) .
$$

This relation is sufficient to show that the path-integral over $\beta(\sigma)$ will be free from 1-loop logarithmic divergences. From the most singilar term we read off the propagator

$$
\langle\beta(p) \beta(-p)\rangle=\frac{1}{2|p|^{3}}
$$

Possible vertices coming from lower order terms in (2.35) will produce integrals with a UV asymptotic behavior of

$$
\int^{\infty} d p \frac{O(p)}{|p|^{3}}
$$

Since this is absolutely convergent, logarithmic divergences will be absent. Again, the same conclusion can be reached by heat-kernel analysis departing from (2.35).

The above derivation resolves the puzzle mentioned in Section 2.4 and explains quantitatively the difference between having the contour on the absolute and inside the AdS. For a contour strictly inside, the dependence of the action on reparametrizations would be of the form $|p|+O\left(p^{0}\right)$ similar to (2.23), and conformal invariance would be broken by logarithmic divergences.

\section{Fixed contours and D0-branes}

As we saw in the previous section, fixed boundary amplitudes in string theory lead naturally to path-integrals over reparametrizations after conformal gauge fixing. These integrals are sure to contain important dynamical information relevant to gauge/string duality, and it is very important to learn how to work with them. 
As a first step in this direction, we are going to perform a detailed analysis of the flat-space integral (2.20). One should not forget of course that our main interest is with the AdS integral (2.31). However, its treatment is greatly complicated by not knowing the action explicitly, and I postpone it to a later occasion. The integral (2.20) has a definite advantage in this respect. We will be able to obtain rather precise statements about it, and along the way learn important general lessons about the nature of reparametrization path-integrals.

\subsection{Do-brane analogy}

The flat-space "Wilson loop" integral (2.20) is formally identical to a path-integral arizing in the $\sigma$-model description of a bosonic D0-brane, when only the fields describing the shape of the brane are turned on. To see this, it is sufficient to rewrite the usual D0-brane boundary conditions

$$
\left.X^{0}\right|_{\tau=0}=\text { free },\left.\quad X^{i}\right|_{\tau=0}=\phi^{i}\left(X^{0}\right) \quad(i=1 \ldots 25)
$$

in the covariant form

$$
X^{\mu}(\sigma, 0)=c^{\mu}(\alpha(\sigma)) .
$$

As a result, we get an integral of exactly the same form as (2.20).

In this approach the only difference between Wilson loops and D0-branes is in the nature of the field $\alpha(\sigma)$. In the Wilson loop path-integral, $\alpha(\sigma)$ is a diffeomorphism so that

$$
\dot{\alpha}(\sigma)>0
$$

However, in the D0-brane case $\alpha(\sigma)$ does not have to satisfy this constraint, because the field $X^{0}$ was free to backtrack. In other words, the worldsheet attached to the D0-brane can have folds. The measure of integration is also different

$$
\begin{aligned}
\|\delta \alpha\|^{2} & =\int d \sigma[\delta \alpha(\sigma)]^{2} & & \text { (D0-brane) } \\
\|\delta \alpha\|^{2} & =\int d \sigma \dot{\alpha}(\sigma)[\delta \alpha(\sigma)]^{2} & & \text { (Wilson loop). }
\end{aligned}
$$

The former is the usual Gaussian measure, while the latter is the measure on Diff $\left(S^{1}\right)$ invariant under right shifts $\alpha(\sigma) \rightarrow \alpha(f(\sigma))$.

The group Diff $\left(S^{1}\right)$ has a boundary consisting of $\alpha(\sigma)$ for which

$$
\dot{\alpha}(\sigma)=0
$$


on an interval. I am quite sure that this boundary affects nonperturbative dynamics, and in particular gives rise to an anomaly in reparametrization Ward identities. However, at present I am unable to demonstrate this quantitatively. On the other hand, I expect and assume for the purposes of this paper that the perturbation theory of small oscillations around a particular $\alpha_{*}(\sigma)$ should not feel the presence of the boundary (3.6).

Admittedly, this assumption has to be looked into more carefully. However, even if it will later be found to be incorrect, this will not invalidate what I have to say below, but rather just restrict it to the D-brane case. Such a discovery of a different perturbative sector in critical string theory seems an exciting however unlikely possibility.

\subsection{Renormalization and D0-brane effective action}

From a practical point of view, the path-integral (2.20) describes a nonlocal quantum field theory of the field $\alpha(\sigma)$. As we saw in Section 2.5, in spite of being 1-dimensional, this field theory is UV divergent (unlike say the usual quantum mechanics).

In Section 2.5 we concluded that if the contour is a straight line, the 1-loop divergence (2.29) is absent. For a general contour this divergence is nonlocal and cannot be removed by local counterterms. However, there is another natural way to deal with it. Namely, since the divergence is proportional to $\delta S_{\mathrm{cl}} / \delta c$, it can be removed by renormalizing the contour

$$
c \rightarrow c+\delta c, \quad \delta c^{\mu}=-\alpha^{\prime} \log \Lambda \frac{\ddot{c}^{\mu}}{\dot{c}^{2}} .
$$

In fact because of (2.25) I can add any multiple of $\dot{c}^{\mu}$ to this formula. It is natural to write this 1-loop RG in the reparametrization-invariant form

$$
\beta^{\mu}(c)=\frac{d c^{\mu}}{d \log \Lambda}=-\alpha^{\prime} \frac{\ddot{c}_{\perp}^{\mu}}{\dot{c}^{2}}, \quad \ddot{c}_{\perp}^{\mu}=\ddot{c}^{\mu}-\dot{c}^{\mu} \frac{(\dot{c} \ddot{c})}{\dot{c}^{2}}
$$

The " $\beta$-function equation"

$$
\beta^{\mu}=0
$$

is equivalent to (2.30) and has straight lines as solutions.

It is well known [24, 5] that string theory world-sheet $\beta$-functions are related to the low energy effective action by a generic formula

$$
\beta^{m}(\phi)=g^{m l}(\phi) \frac{\delta \Gamma(\phi)}{\delta \phi^{l}}
$$

where $g^{m l}(\phi)$ is a nondegenerate metric in the space of massless space-time fields $\phi$..

A direct way to compute $\Gamma$ would be to calculate $S$-matrix amplitudes and construct a space-time action which reproduces them. Formula (3.10) says that instead we may 
consider the nonlinear $\sigma$-model describing a string propagating in background fields $\phi$. Conditions of conformal invariance of this $\sigma$-model will coincide with equations of motion for $\Gamma$. This truly remarkable equivalence holds in all known cases, although a general proof to the best of my knowledge has not been given.

The advantage of the second method is that it produces a manifestly covariant derivative expansion of $\Gamma$. To obtain higher and higher order in $\alpha^{\prime}$ terms in this expansion, one just has to compute the $\sigma$-model $\beta$-function to more and more loops.

It is reassuring to see that our RG (3.8) can be rewritten in the form of (3.10) as

$$
\beta^{\mu}(c)=-\frac{\alpha^{\prime}}{\sqrt{\dot{c}^{2}}} \frac{\delta S_{\mathrm{eff}}}{\delta c^{\mu}(\sigma)}
$$

where

$$
S_{\text {eff }}=\int d \sigma \sqrt{\dot{c}^{2}}
$$

Thus we recover the usual bosonic D0-brane effective action (in the particular background $G_{\mu \nu}=\eta_{\mu \nu}, B_{\mu \nu}=0, \Phi=$ const). We see that the reparametrization path-integral provides a convenient framework for obtaining this classical result.

While the 1-loop calculation can be actually done directly on the world-sheet without invoking the non-local action (2.19) explicitly (see [25]), the representation (2.20) becomes essential when we move beyond one loop, as we do below.

\subsection{Renormalizability beyond one loop}

Let me repeat the logic of the above discussion. We considered a 1-dimensional QFT with partition function (2.20), and found that it is 1-loop renormalizable. At first this may seem like a pure coincidence, since the theory is non-local, and no general renormalizability arguments apply to it. However, the relation with D0-brane dynamics expressed by (3.11) suggests that this is not so. In fact, I conjecture that renormalizability must hold to any loop order. Moreover, the $\alpha^{\prime}$ expansion of the $\beta$-function must have the form following from $(3.10)$

$$
\beta^{\mu}(c)=g^{\mu \nu}(c) \frac{\delta S_{\mathrm{eff}}}{\delta c^{\nu}(\sigma)} .
$$

The metric $g^{\mu \nu}(c)$ may and will in general get $\alpha^{\prime}$ corrections compared to (3.11)

$$
g^{\mu \nu}(c)=-\frac{\alpha^{\prime}}{\sqrt{\dot{c}^{2}}} \delta^{\mu \nu}+\ldots
$$

As for the $S_{\text {eff }}$, the only possible covariant corrections must be constructed from higher order derivatives with respect to the natural parameter $s$, such as the curvature $c_{s s}^{\prime \prime}$, torsion 
$c_{s s s}^{\prime \prime \prime}$, etc. However, all these terms would vanish on shell, that is for straight lines. Thus we arrive at the conclusion that the effective action (3.12) should not get any perturbative corrections.

Two-loop computations that I do below confirm these predictions about renormalizability and the form of the $\beta$-function.

\subsection{Circular contour}

Before we tackle 2-loop corrections for a general contour, it is instructive to consider the example when $C$ is a circle

$$
\begin{aligned}
& c^{0}(\sigma)=R \cos \sigma, \\
& c^{1}(\sigma)=R \sin \sigma \\
& c^{i}(\sigma)=0 \quad(i=2 \ldots 25) .
\end{aligned}
$$

In this case the only renormalizable parameter is the radius $R$. It is convenient to include it into the definition of the coupling constant $g$

$$
\frac{1}{g}=\frac{R^{2}}{4 \pi \alpha^{\prime}}
$$

Because we use $0<\sigma<2 \pi$ to parametrize the circle, the classical action (2.19) changes to the discrete sum

$$
S_{\mathrm{cl}}=\frac{1}{2 \pi} \sum_{p \in \mathbb{Z}}|p| c_{p}^{\mu} c_{-p}^{\mu}
$$

The action $S_{\mathrm{cl}}\left[c^{\mu}(\alpha(\sigma))\right]$ is easily found by using the representation

$$
c^{\mu}(\alpha(\sigma)) c^{\mu}\left(\alpha\left(\sigma^{\prime}\right)\right)=\operatorname{Re} \exp i\left(\alpha(\sigma)-\alpha\left(\sigma^{\prime}\right)\right) .
$$

It is given by

$$
S_{\mathrm{cl}}\left[c^{\mu}(s+\beta(\sigma))\right]=\frac{1}{4 \pi} \sum_{p \in \mathbb{Z}}(|p+1|+|p-1|) \sum_{k, l \geq 0} \frac{i^{k-l}}{k ! l !}\left(\beta^{k}\right)_{p}\left(\beta^{l}\right)_{-p}
$$

It is easy to see that all odd order terms vanish in this action. In particular, the linear term in $\beta$ is absent. The quadratic action is given by

$$
S_{2}=\frac{1}{4 \pi} \sum E(p) \beta_{p} \beta_{-p}, \quad E(p)=|p+1|+|p-1|-2
$$


It should come as no surprise that $E(p)=0$ for $p=0, \pm 1$. These three zero modes reflect the $S L(2, \mathbb{R})$ invariance of $S_{\mathrm{cl}}$.

We view (2.20) as a non-local quantum theory of the field $\beta(\sigma)$ with the classical action (3.19). We expect that all correlators of this theory can be made finite by adding counterterms to the action renormalizing the coupling constant $g$. Below I will show that this is indeed true for all 4-point functions in the 1-loop order, and for all 2-point functions in the 2-loop order. We will also find the 2-loop $\beta$-function.

To perform this calculation, we will need the first two higher order terms in (3.19)

$$
\begin{aligned}
& S_{4}=\frac{1}{2 \pi} \sum E(p)\left(\frac{1}{8}\left(\beta^{2}\right)_{p}\left(\beta^{2}\right)_{-p}-\frac{1}{6} \beta_{p}\left(\beta^{3}\right)_{-p}\right), \\
& S_{6}=\frac{1}{2 \pi} \sum E(p)\left(\frac{1}{120} \beta_{p}\left(\beta^{5}\right)_{-p}-\frac{1}{48}\left(\beta^{2}\right)_{p}\left(\beta^{4}\right)_{-p}+\frac{1}{72}\left(\beta^{3}\right)_{p}\left(\beta^{3}\right)_{-p}\right) .
\end{aligned}
$$

Denoting the nonlocal $E(p)$ vertex by a wavy line, we have the following diagrammatic representation

$$
\begin{aligned}
S & =S_{2}+S_{4}+S_{6}+\ldots \\
& =\frac{1}{2} \leadsto+\left(\frac{1}{8} \leadsto<-\frac{1}{6} \sim \ldots k\right) \\
& +\left(\frac{1}{120} \sim m k-\frac{1}{48}>n k+\frac{1}{72}>n<\right)+\ldots .
\end{aligned}
$$

Contracting these diagrams with the propagator $E(p)^{-1}$, we obtain the 1-loop correction to the quantum effective action through $\beta^{4}$ terms

$$
\begin{aligned}
& S_{1-\text { loop }}=g\left(\frac{1}{2} \smile-\frac{1}{2} \ngtr y-\frac{1}{2} \ln \right. \\
& +\frac{1}{12} \ln k-\frac{1}{8} \ln k+\frac{1}{12} \text { nk } \\
& \left.+\frac{1}{24} \text { yk }-\frac{1}{6}-w k+\frac{1}{8} \mathfrak{y}\right)+\ldots
\end{aligned}
$$

Notice that the diagrams like $2 m 0$ vanish, because $E(0)=0$. The change in the numerical factors reflects the numbers of equivalent contractions.

The r.h.s. of (3.23) is divergent. First of all, it contains logarithmic divergences proportional to the "leaf" diagram

$$
\mathrm{O}=\frac{1}{2 \pi} \sum_{|p|<\Lambda}^{\prime} \frac{1}{E(p)}=\frac{1}{2 \pi} \log \Lambda+\text { finite }
$$

where $\sum^{\prime}$ is being taken over $p \neq 0, \pm 1$. Superficially, it also contains linear divergences proportional to the "oyster" $r$. 
However, as the reader may easily check, the "oyster" divergences of individual diagrams are local and cancel each other when added (for instance, in the last line of (3.23) this happens because $1 / 24-1 / 6+1 / 8=0$ ). What remains is the overall logarithmic divergence, and it is easy to see that it is exactly proportional to the $S_{2}+S_{4}$ part of the classical action. It can be removed by adding the counterterm

$$
S_{c t}=\left(\frac{g}{2 \pi} \log \Lambda\right) S
$$

which translates into the RG law for the bare coupling constant

$$
\begin{aligned}
& \frac{1}{g(\Lambda)}=\frac{1}{g}+\frac{1}{2 \pi} \log \Lambda, \\
& \beta(g)=\frac{d g}{d \log \Lambda}=-\frac{g^{2}}{2 \pi} .
\end{aligned}
$$

A simple check shows that this 1-loop $\beta$-function agrees with the general contour result (3.8).

It is interesting to note that the partition function renormalization of Section 3.2 and the above correlator renormalization follow in fact from two quite different and formally inequivalent computations. Their agreement should not be taken as a pure coincidence. Rather, it is a sign of a renormalizable field theory structure hidden behind.

Let us now discuss order $g^{2}$ corrections to the quantum effective action. In this order 2-loop terms coming from the original expansion (3.22) mix with contributions of 1-loop counterterms. The quadratic part of the effective action consists of the following diagrams
(a) $-\sqrt{S_{6}}-\frac{1}{2}-S_{4}-S_{4}$
(b) $-\frac{1}{2}-S_{4} \boxminus S_{4}$
(c) $-\widehat{S_{4}^{c t}}--S_{4}-S_{2}^{c t}$
(d) $-\frac{1}{2}-S_{2}^{c t}-S_{2}^{c t}-$

It is quite easy to see directly that the divergent parts of the diagrams in (c) cancel identically. The same happens for (b), although in a subtler way: the diagrams fall into groups, and within each group divergences cancel. For instance, one of the groups is

$$
\frac{1}{2} \xi \xi-\frac{1}{4} \hat{\xi}-\frac{1}{4} \xi \xi<\infty \text {. }
$$

Class (a) is the most numerous one, it consists of about 20 diagrams. Again many cancellations similar to (3.29) occur. In the end all that remains are two diagrams from 
the first term

$$
\frac{1}{8} \ln h+\frac{1}{8} \text { inf }
$$

and three diagrams from the second one

$$
\frac{1}{2}-y-\frac{1}{2} \text { wy }-\frac{1}{2} \text { y. }
$$

The only nontrivial calculation is required for the divergence

$$
\begin{aligned}
\triangleq-\circledast 1 & =\frac{1}{(2 \pi)^{2}} \sum_{|q|,|p|<\Lambda}^{\prime} \frac{E(q-p)-E(q)}{E(p)^{2} E(q)} \\
& =\frac{1}{8 \pi^{2}}(\log \Lambda)^{2}+\frac{1}{4 \pi^{2}}(\gamma-1) \log \Lambda+\text { finite },
\end{aligned}
$$

where the Euler constant $\gamma$ appears as the finite part in the harmonic series

$$
\sum_{n=1}^{\Lambda} \frac{1}{n}=\log \Lambda+\gamma+O\left(\Lambda^{-1}\right) .
$$

Adding divergences in (3.28), (3.30), and (3.31), we get the total order $g^{2}$ divergent contribution to the quadratic effective action equal to

$$
\sim-\left(-\frac{g^{2}}{16 \pi^{2}}(\log \Lambda)^{2}-\frac{g^{2}}{8 \pi^{2}} \log \Lambda\right) .
$$

Remarkably, the finite part $\gamma$ canceled in this final result. As we will see in Section 3.5 below, a similar cancellation of finite parts of divergent Green's functions occurs on a much larger scale for general contours, and in fact is essential for the existence of local counterterms.

Being proportional to $S_{2}$, the divergence (3.34) can again be canceled by a counterterm. This requires the following $O(g)$ correction to (3.26)

$$
\frac{1}{g(\Lambda)}=\frac{1}{g}+\frac{1}{2 \pi} \log \Lambda+\frac{g}{8 \pi^{2}}(\log \Lambda)^{2}+\frac{g}{4 \pi^{2}} \log \Lambda .
$$

An important check of the general structure is provided by the $(\log \Lambda)^{2}$ term in this expression: its value agrees with the one following from the 1-loop $\beta$-function as dictated by renormalizability. The subleading $g \log \Lambda$ term corrects the $\beta$-function

$$
\beta(g)=-\frac{g^{2}}{2 \pi}-\frac{g^{3}}{4 \pi^{2}} .
$$


Thus we see that to the order that we were able to compute, the circular contour reparametrization path-integral does indeed define a renormalizable quantum theory. I do not know if a simple proof of renormalizability to all orders can be given along the lines of the above argument. Perhaps one may use $S L(2, \mathbb{R})$ invariance to restrict the form of the quantum action. At present this has not been done.

In the next section, we will renormalize the path-integral (2.20) for a general contour, finding a 2-loop $\beta$-function agreeing with (3.36). This will provide yet another check for our claim of renormalizability.

\subsection{Two-loop renormalization for a general contour}

To compute 2-loop corrections in (2.20), we must expand the action to the 4 th order in $\beta$. It is convenient (leads to considerable simplifications and manifest covariance) to perform this expansion in terms of the "normal coordinate" field $y(\sigma)$ defined as the invariant length between $c_{\mu}(\sigma)$ and $c_{\mu}(\sigma+\beta(\sigma))$

$$
y(\sigma)=\int_{\sigma}^{\sigma+\beta(\sigma)} \sqrt{\dot{c}^{2}} .
$$

In terms of $y(\sigma)$ we have the Taylor expansion

$$
c_{\mu}(\sigma+\beta(\sigma))=\sum \frac{1}{k !} \frac{d^{k} c_{\mu}}{d s^{k}}(\sigma) y^{k}
$$

where $s$ is the natural parameter (the $s$ derivatives are denoted below by a prime). Substituting this into (2.22), we get

$$
S=S_{\mathrm{cl}}\left[c_{\mu}(\sigma)\right]+S_{2}+S_{3}+S_{4}+\ldots
$$

with

$$
\begin{aligned}
S_{2} & =\iint d \sigma d \sigma^{\prime} K_{\sigma \sigma^{\prime}} c_{\mu}^{\prime} y(\sigma) c_{\mu}^{\prime} y\left(\sigma^{\prime}\right)+\int d \sigma\left(\psi c^{\prime \prime}\right) y^{2}, \\
S_{3} & =\iint K_{\sigma \sigma^{\prime}} c_{\mu}^{\prime} y(\sigma) c_{\mu}^{\prime \prime} y^{2}\left(\sigma^{\prime}\right)+\frac{1}{3} \int\left(\psi c^{\prime \prime \prime}\right) y^{3}, \\
S_{4} & =\frac{1}{4} \iint K_{\sigma \sigma^{\prime}} c_{\mu}^{\prime \prime} y^{2}(\sigma) c_{\mu}^{\prime \prime} y^{2}\left(\sigma^{\prime}\right) \\
& +\frac{1}{3} \iint K_{\sigma \sigma^{\prime}} c_{\mu}^{\prime} y(\sigma) c_{\mu}^{\prime \prime \prime} y^{3}\left(\sigma^{\prime}\right)+\frac{1}{12} \int\left(\psi c^{\prime \prime \prime \prime}\right) y^{4} .
\end{aligned}
$$


Here we denoted

$$
K_{\sigma \sigma^{\prime}}=-\frac{1}{\pi\left(\sigma-\sigma^{\prime}\right)^{2}}, \quad \psi_{\mu}=\frac{1}{2} \frac{\delta S_{\mathrm{cl}}}{\delta c_{\mu}} .
$$

The path-integral (2.20) can now be computed perturbatively using Feynman rules. The propagator

$$
G\left(\sigma, \sigma^{\prime}\right)=\int[\mathcal{D} y] y(\sigma) y\left(\sigma^{\prime}\right) \exp \left(-S_{2}\right)
$$

cannot in general be found explicitly. Being the Green's function of the quadratic action, it satisfies the integral equation

$$
c_{\mu}^{\prime}(\sigma) \int d \sigma^{\prime} K_{\sigma \sigma^{\prime}} c_{\mu}^{\prime}\left(\sigma^{\prime}\right) G\left(\sigma^{\prime}, \sigma^{\prime \prime}\right)+\left(\psi c^{\prime \prime}\right)(\sigma) G\left(\sigma, \sigma^{\prime \prime}\right)=\frac{1}{2} \delta\left(\sigma-\sigma^{\prime \prime}\right) .
$$

In the 1-loop approximation the effective action $F=-\log Z$ is given by

$$
F=\frac{1}{4 \pi \alpha^{\prime}} S_{\mathrm{cl}}[c]+\frac{1}{2} \bigcirc .
$$

As we discussed in Section 2.5, the log-determinant is logarithmically divergent护

$$
\frac{1}{2} \bigcirc=\frac{1}{2 \pi} \log \Lambda \int\left(\psi c^{\prime \prime}\right)+\text { finite } .
$$

To cancel this divergence, in Section 3.2 we added a counterterm renormalizing the contour

$$
\delta c_{\mu}=-\alpha^{\prime} \log \Lambda c_{\mu}^{\prime \prime} .
$$

Now we would like to move further and consider 2-loop corrections. In this order the 1-particle irreducible effective action is given by

$$
F_{1 \mathrm{PI}}=\frac{1}{4 \pi \alpha^{\prime}} S_{\mathrm{cl}}[c+\delta c]+\frac{1}{2} \bigcirc+\delta S_{2}+4 \pi \alpha^{\prime}\left(\widehat{S_{4}}-\frac{1}{2} S_{3}=\widehat{S_{3}}\right) .
$$

The change in $S_{2}$ due to the 1-loop counterterm is

$$
\delta S_{2}=2 \iint K_{\sigma \sigma^{\prime}} \delta c_{\mu}^{\prime} y(\sigma) c_{\mu}^{\prime} y\left(\sigma^{\prime}\right)+\int\left(\delta \psi_{\mu} c_{\mu}^{\prime \prime}+\psi_{\mu} \delta c_{\mu}^{\prime \prime}\right) y^{2},
$$

\footnotetext{
${ }^{5}$ Strictly speaking, there is also a linear divergence. But it is contour independent and can be ignored.
} 
where

$$
\begin{aligned}
\delta \psi_{\mu}(\sigma) & =-\alpha^{\prime} \log \Lambda \int d \sigma^{\prime} K_{\sigma \sigma^{\prime}} c_{\mu}^{\prime \prime}\left(\sigma^{\prime}\right), \\
\delta\left(c_{\mu}^{\prime}\right) & =-\alpha^{\prime} \log \Lambda\left(c_{\mu}^{\prime \prime \prime}-c_{\mu}^{\prime}\left(c^{\prime} c^{\prime \prime \prime}\right)\right) \\
\delta\left(c_{\mu}^{\prime \prime}\right) & =-\alpha^{\prime} \log \Lambda\left(c_{\mu}^{\prime \prime \prime \prime}-2 c_{\mu}^{\prime \prime} c^{\prime \prime 2}-c_{\mu}^{\prime}\left(c^{\prime} c^{\prime \prime \prime}\right)^{\prime}\right) .
\end{aligned}
$$

We have to analyze divergences in the following diagrams

$$
\begin{aligned}
& \delta S_{2}=\cdots r+\cdots
\end{aligned}
$$

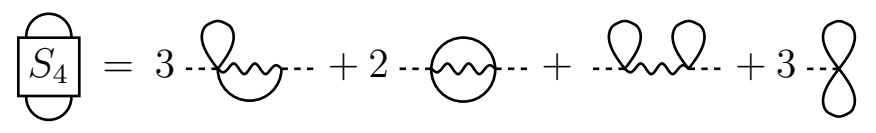

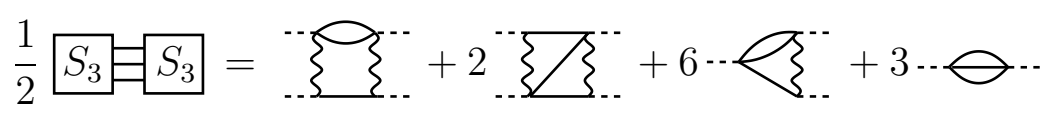

Here $-=G\left(\sigma, \sigma^{\prime}\right)$, $\sim=K_{\sigma \sigma^{\prime}}$, and the dashed lines denote the $\sigma$-dependent coefficients present in (3.40) and (3.48). The numerical factors account for equivalent contractions.

Consider as an example the first diagram in (3.51), corresponding to the integral

$$
\operatorname{lng}_{--}=\frac{1}{3} \iint K_{\sigma \sigma^{\prime}} c_{\mu}^{\prime}(\sigma) c_{\mu}^{\prime \prime \prime}\left(\sigma^{\prime}\right) G\left(\sigma, \sigma^{\prime}\right) G\left(\sigma^{\prime}, \sigma^{\prime}\right)
$$

An immediate source of infinities in this expresssion is the presence of $G\left(\sigma^{\prime}, \sigma^{\prime}\right)$ in the integrand (the corresponding part of the diagram is the leaf $\mathrm{O}$ ). The regulated Green's function at coincident points is logarithmically divergent

$$
G(\sigma, \sigma)=\frac{1}{2 \pi} \log \Lambda+G_{\text {fin }}(\sigma)
$$

In fact, the propagator has the following asymptotic expansion

$$
\begin{aligned}
& G(\sigma, p)=\frac{1}{2|p|}-\frac{\left(\psi c^{\prime \prime}\right)(\sigma)}{2 p^{2}}+\ldots \quad(p \rightarrow \infty), \\
& G(\sigma, p) \stackrel{\text { def }}{=} \int G\left(\sigma+\frac{h}{2}, \sigma-\frac{h}{2}\right) e^{i p h} d h .
\end{aligned}
$$

This formula is not difficult to obtain from (3.43). Eq. (3.54) follows from (3.55) with an unknown finite part which I denoted $G_{\text {fin }}(\sigma)$. 
However, the leaf is not the only source of divergence in (3.53). A separate infinity comes from the oyster

$$
\oiiint \sim \iint K_{\sigma \sigma^{\prime}} G\left(\sigma, \sigma^{\prime}\right)
$$

This divergence is equal to

$$
\int \frac{d p}{2 \pi}|p| G(\sigma, p)=-\frac{1}{2 \pi} \log \Lambda\left(\psi c^{\prime \prime}\right)(\sigma)+\text { finite }
$$

(ignoring a term linear in $\Lambda$ ).

Notice that the divergent parts of (3.54) and (3.57) are known exactly. On the contrary, the finite parts, like $G_{\text {fin }}(\sigma)$ in (3.54), depend on the data in some complicated nonlocal way and cannot in general be found explicitly. However, the total divergence of (3.53) will arise as the product of (3.54) and (3.57). So in addition to the explicitly known $(\log \Lambda)^{2}$ terms it will contain subleading $\log \Lambda$ cross-terms proportional to unknown finite parts. This presents a potentially serious problem for renormalizability, because at the end of the calculation I expect to obtain a local counterterm.

Similar problems with finite parts appear in renormalizing local field theories, such as $\lambda \phi^{4}$ theory, in curved space-time. There it was found [26] that divergent contributions containing finite parts cancel when individual diagrams are added. We will see below that such a cancellation happens in our case as well.

Careful analysis of divergences in 2-loop diagrams is carried out in Appendix B along the lines of the above discussion. The only nontrivial calculations are required for the following three diagrams

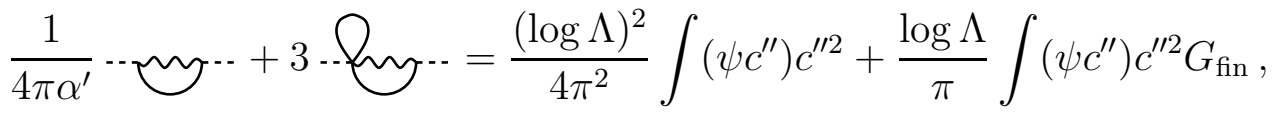

$$
\begin{aligned}
& \left.2 \ldots-1-\frac{(\log \Lambda)^{2}}{16 \pi^{2}}+\frac{\log \Lambda}{8 \pi^{2}}\right) \int\left(\psi c^{\prime \prime}\right) c^{\prime \prime 2}-\frac{\log \Lambda}{4 \pi} \int\left(\psi c^{\prime \prime}\right) c^{\prime \prime 2} G_{\text {fin }}
\end{aligned}
$$

(it turns out convenient to combine the first two). The diagrams in (3.52) are shown to be convergent. All the remaining diagrams do not contain oysters and their divergences are very easy to write down using (3.54). For example

$$
\text { - hn . }=\frac{(\log \Lambda)^{2}}{16 \pi^{2}} \iint K_{\sigma \sigma^{\prime}} c_{\mu}^{\prime \prime}(\sigma) c_{\mu}^{\prime \prime}\left(\sigma^{\prime}\right)+\frac{\log \Lambda}{4 \pi} \iint K_{\sigma \sigma^{\prime}} G_{\text {fin }}(\sigma) c_{\mu}^{\prime \prime}(\sigma) c_{\mu}^{\prime \prime}\left(\sigma^{\prime}\right)+\text { finite }
$$

We should not also forget about the order $\alpha^{\prime}$ term

$$
\frac{1}{4 \pi \alpha^{\prime}} \iint K_{\sigma \sigma^{\prime}} \delta c_{\mu}(\sigma) \delta c_{\mu}\left(\sigma^{\prime}\right)
$$


coming from expanding the classical action in (3.47).

I am now going to report the result of these elementary calculations. The total order $\alpha^{\prime}$ divergence in (3.47) comes out to be equal

$$
\alpha^{\prime} \int \frac{(\log \Lambda)^{2}}{4 \pi}\left(-\left(\psi c^{\prime \prime \prime \prime}\right)+2\left(\psi c^{\prime \prime}\right) c^{\prime \prime 2}\right)+\frac{\log \Lambda}{\pi}\left(\psi c^{\prime \prime}\right) c^{\prime \prime 2} .
$$

In particular, all the terms proportional to $G_{\text {fin }}$ indeed cancel identically in this final result, and the above-mentioned problem happily resolves itself. Moreover, since the overall divergence is again proportional to $\psi_{\mu}$, it can be removed by adding an $\alpha^{\prime 2}$ correction to the 1-loop counterterm. The total 2-loop counterterm is equal to

$$
\delta c_{\mu}=-\alpha^{\prime} \log \Lambda c_{\mu}^{\prime \prime}+\frac{1}{2} \alpha^{\prime 2}(\log \Lambda)^{2}\left(c_{\mu}^{\prime \prime \prime \prime}-2 c_{\mu}^{\prime \prime} c^{\prime \prime 2}-c_{\mu}^{\prime}\left(c^{\prime} c^{\prime \prime \prime}\right)^{\prime}\right)-2 \alpha^{2} \log \Lambda c_{\mu}^{\prime \prime} c^{\prime \prime 2}
$$

Notice that renormalizability requires that the $(\log \Lambda)^{2}$ coefficient be expressed via the 1-loop $\beta$-function as

$$
\frac{1}{2} \int d \sigma^{\prime} \frac{\delta \beta^{\mu}(\sigma)}{\delta c_{\nu}\left(\sigma^{\prime}\right)} \beta^{\nu}\left(\sigma^{\prime}\right) .
$$

To put the coefficient in (3.63) in agreement with this formula, we added a term proportional to $c_{\mu}^{\prime}$. This new term does not affect cancellation of divergences, because $\left(\psi c^{\prime}\right) \equiv 0$.

The $\alpha^{\prime 2}$ correction to the $\beta$-function is given by the $\alpha^{\prime 2} \log \Lambda$ coefficient

$$
\beta^{\mu}(c)=-\left(\alpha^{\prime}+2 \alpha^{\prime 2} c^{\prime \prime 2}\right) c_{\mu}^{\prime \prime}
$$

This formula is the main result of this section. It is not hard to see that it agrees with the circular contour 2-loop $\beta$-function (3.36).

This concludes our analysis of the reparametrization path-integral (2.20). Obviously we are leaving many questions open, most notably nonperturbative effects and the precise relation between the D0-brane and the Wilson loop case (see Section 3.1). I plan to return to these matters in a future publication.

\section{Reparametrization path-integrals and $\mathrm{D} p$-brane dynamics}




\subsection{Nonlocal field theory on the boundary}

The basic conclusion of the previous section was that the D0-brane equations of motion arise naturally from studying logarithmic divergences in the reparametrization pathintegral (2.20).

I would now like to consider a generalization of this idea to the case of $\mathrm{D} p$-branes. The $\mathrm{D} p$-brane boundary conditions can be written covariantly as

$$
X^{\mu}(\sigma, 0)=F^{\mu}\left(x^{0}(\sigma), \ldots, x^{p}(\sigma)\right) \quad(\mu=0 \ldots 25) .
$$

Here the functions $F^{\mu}\left(x^{a}\right)$ describe the shape of the brane, while the $p+1$ fields $x^{a}(\sigma)$ realize the free boundary conditions for $X^{a}$. This is all analogous to (3.2), except that instead of reparametrizations we are now dealing with maps from $S^{1}$ into $\mathbb{R}^{p+1}$.

The corresponding path-integral generalizing $(2.20)$ is

$$
\begin{aligned}
& \int\left[\mathcal{D} x^{a}(\sigma)\right] \exp \left(-\frac{1}{4 \pi \alpha^{\prime}} S\right), \\
& S=\iint K_{\sigma \sigma^{\prime}} F^{\mu}(x(\sigma)) F^{\mu}\left(x\left(\sigma^{\prime}\right)\right) .
\end{aligned}
$$

From our experience with (2.20), we expect that the $d=1$ theory of $p+1$ fields $x^{a}(\sigma)$ described by this integral is going to be renormalizable by adding counterterms changing the shape of the brane. This is indeed true to the extent that I was able to check it. Here I am going to present a computation that is technically slightly simpler than demonstrating renormalizability to the same scope as I did it for (2.20). Namely, we will find a geometric condition on $F^{\mu}$ for the cancellation of 2-loop divergences in (4.2). This will give us the $\mathrm{D} p$-brane effective action including the first $\alpha^{\prime}$ correction. This is of particular interest, since unlike in the D0-brane case, the correction will be nontrivial. We will then compare the result of this calculation with the effective action computed directly from the $S$-matrix amplitudes, finding complete agreement.

\subsection{Covariant perturbation theory}

I would like to develop a perturbative expansion of (4.2). To begin with, I choose a configuration of fields $\bar{x}^{a}(\sigma)$ providing a minimum to the action [o To keep things explicitly covariant, I am going to use Riemann normal coordinates with respect to the induced metric

$$
G_{a b}=\partial_{a} F^{\mu} \partial_{b} F^{\mu} .
$$

\footnotetext{
${ }^{6}$ To ensure that such a configuration exists, I can assume that the brane is asymptotically flat at infinity and impose the "long string" boundary conditions $x^{a}(\sigma) \rightarrow \pm \infty$ as $\sigma \rightarrow \pm \infty$. The action of such a long string coincides with (4.3) up to an irrelevant infinite constant.
} 
For each $\sigma$, let $y^{a}(\sigma)$ be such coordinates near $\bar{x}(\sigma)$. I use the normal coordinate field $y^{a}(\sigma)$ to represent the field $x(\sigma)$, which is supposed to be close to $\bar{x}(\sigma)$. In these coordinates we have the expansion (see e.g. [27])

$$
F^{\mu}(x(\sigma))=F^{\mu}(\bar{x}(\sigma))+\bar{E}_{a}^{\mu} y^{a}+\frac{1}{2} \bar{\Omega}_{a b}^{\mu} y^{a} y^{b}+\frac{1}{6} \bar{L}_{a b c}^{\mu} y^{a} y^{b} y^{c}+\frac{1}{24} \bar{M}_{a b c d}^{\mu} y^{a} y^{b} y^{c} y^{d}+\ldots
$$

where

$$
\begin{array}{cl}
\bar{E}_{a}^{\mu}=\partial_{a} F^{\mu}(\bar{x}(\sigma)), & \bar{L}_{a b c}^{\mu}=\nabla_{(a} \nabla_{b} \partial_{c)} F^{\mu}(\bar{x}(\sigma)), \\
\bar{\Omega}_{a b}^{\mu}=\nabla_{a} \partial_{b} F^{\mu}(\bar{x}(\sigma)), & \bar{M}_{a b c d}^{\mu}=\nabla_{(a} \nabla_{b} \nabla_{c} \partial_{d)} F^{\mu}(\bar{x}(\sigma)) .
\end{array}
$$

For future reference note that

$$
\left(\bar{E}_{a} \bar{E}_{b}\right)=\delta_{a b},
$$

since we are in normal coordinates.

Substituting this expansion into (4.3), we get analogously to Section 3.5

$$
\begin{aligned}
S & =S[\bar{x}(\sigma)]+S_{2}+S_{3}+S_{4}+\ldots, \\
S_{2} & =\iint K_{\sigma \sigma^{\prime}} \bar{E}_{a}^{\mu} y^{a}(\sigma) \bar{E}_{b}^{\mu} y^{b}\left(\sigma^{\prime}\right)+\int\left(\bar{\Psi} \bar{\Omega}_{a b}\right) y^{a} y^{b}, \\
S_{3} & =\iint K_{\sigma \sigma^{\prime}} \bar{E}_{a}^{\mu} y^{a}(\sigma) \bar{\Omega}_{b c}^{\mu} y^{b} y^{c}\left(\sigma^{\prime}\right)+\frac{1}{3} \int\left(\bar{\Psi} \bar{L}_{a b c}\right) y^{a} y^{b} y^{c}, \\
S_{4} & =\frac{1}{4} \iint K_{\sigma \sigma^{\prime}} \bar{\Omega}_{a b}^{\mu} y^{a} y^{b}(\sigma) \bar{\Omega}_{c d}^{\mu} y^{c} y^{d}\left(\sigma^{\prime}\right) \\
& +\frac{1}{3} \iint K_{\sigma \sigma^{\prime}} \bar{E}_{a}^{\mu} y^{a}(\sigma) \bar{L}_{b c d}^{\mu} y^{b} y^{c} y^{d}\left(\sigma^{\prime}\right)+\frac{1}{12} \int\left(\bar{\Psi}_{M_{a b c d}}\right) y^{a} y^{b} y^{c} y^{d},
\end{aligned}
$$

where

$$
\bar{\Psi}^{\mu}(\sigma)=\int d \sigma^{\prime} K_{\sigma \sigma^{\prime}} F^{\mu}\left(\bar{x}\left(\sigma^{\prime}\right)\right), \quad(\bar{\Psi} \bar{E}) \equiv 0 .
$$

The second order action determines the propagator $G^{a b}\left(\sigma, \sigma^{\prime}\right)$. The analogues of (3.54), (3.55) are true

$$
\begin{aligned}
G^{a b}(\sigma, p) & =\frac{\delta^{a b}}{2|p|}-\frac{\left(\bar{\Psi} \bar{\Omega}_{a b}\right)(\sigma)}{2 p^{2}}+\ldots, \\
G^{a b}(\sigma, \sigma) & =\frac{1}{2 \pi} \log \Lambda \delta^{a b}+G_{\text {fin }}^{a b} .
\end{aligned}
$$




\subsection{Analysis of divergences}

The 1-loop divergence in (4.2) is easy to find and is given by

$$
\frac{1}{2} \bigcirc=\frac{1}{2 \pi} \log \Lambda \int\left(\bar{\Psi} \bar{\Omega}_{a a}\right) .
$$

If we want to preserve conformal invariance, this divergence has to cancel, which requires that the trace of the second fundamental form should vanish to this order

$$
\bar{\Omega}_{a a}^{\mu}=O\left(\alpha^{\prime}\right) .
$$

More precisely, this is the most general local condition on the shape of the brane for which the divergence disappears. It is gratifying to see (4.13) arise, because this is exactly the minimal surface equation of motion following from the standard D-brane low energy effective action [25]

$$
\int d^{p+1} x \sqrt{G}
$$

Let me now impose Eq. (4.13), so that there are no order $\alpha^{\prime 0}$ divergences in (4.2). This does not mean of course that there will be no $\alpha^{\prime}, \alpha^{2}$ etc. divergences. In fact, canceling the divergences to higher and higher order in $\alpha^{\prime}$ requires higher and higher order corrections to the equation of motion. Here I am only looking for the first correction. In this order the 1PI effective action is given by (3.47), except that in our present situation there is no counterterm $\delta c$, and $\delta S_{2}$ is also absent. Thus our job will be simpler than in Section 3.5.

Once again we have to find divergent parts of the diagrams (3.51) and (3.52). This is done similarly to the D0-brane calculations from Section 3.5 and Appendix B. Just as before, all the diagrams in (3.52) will be convergent, because of the condition

$$
\left(\bar{E}_{a} \bar{\Omega}_{b c}\right) \equiv 0 .
$$

Analysis of the remaining diagrams is somewhat simplified by the fact that we only need to know the divergences to order $\alpha^{\prime}$. We can use the 1-loop condition (4.13) to show that many terms do not contribute to this order. To give an example of how this happens, consider the third diagram in (3.51)

$$
\text { . } \mathrm{hu} \text {. }=\frac{1}{4} \iint K_{\sigma \sigma^{\prime}} \bar{\Omega}_{a b}^{\mu}(\sigma) G^{a b}(\sigma, \sigma) \bar{\Omega}_{c d}^{\mu}\left(\sigma^{\prime}\right) G^{c d}\left(\sigma^{\prime}, \sigma^{\prime}\right) \text {. }
$$

Because of (4.11), all divergences of this diagram will involve terms like $\bar{\Omega}_{a b}^{\mu} \delta^{a b}$, which are $O\left(\alpha^{\prime}\right)$ by (4.13). So this diagram is irrelevant. 
The logarithmically divergent parts of the other three diagrams in (3.51) are

$$
\begin{aligned}
& 2-\curvearrowleft-1\left(\frac{(\log \Lambda)^{2}}{8 \pi^{2}}-\frac{\log \Lambda}{4 \pi^{2}}\right) \int\left(\bar{\Psi} \bar{\Omega}_{a b}\right) \bar{R}_{a b} \\
& -\frac{\log \Lambda}{2 \pi} \int\left(\bar{\Psi} \bar{\Omega}_{a b}\right) \bar{\Omega}_{a c}^{\mu} \bar{\Omega}_{b d}^{\mu} G_{\text {fin }}^{c d}+O\left(\alpha^{\prime}\right) \\
& 3 \text { - }-1 \text { - }=-\frac{(\log \Lambda)^{2}}{6 \pi^{2}} \int\left(\bar{\Psi} \bar{\Omega}_{a b}\right) \bar{R}_{a b}-\frac{\log \Lambda}{3 \pi} \int\left(\bar{\Psi} \bar{\Omega}_{a b}\right) \bar{R}_{a c} G_{\text {fin }}^{b c} \\
& +\frac{\log \Lambda}{6 \pi} \int\left(\bar{\Psi} \bar{\Omega}_{a b}\right) G_{\text {fin }}^{c d}\left(2 \bar{\Omega}_{a c}^{\mu} \bar{\Omega}_{b d}^{\mu}+\bar{\Omega}_{a b}^{\mu} \bar{\Omega}_{c d}^{\mu}\right)+O\left(\alpha^{\prime}\right) \\
& 3=\frac{(\log \Lambda)^{2}}{24 \pi^{2}} \int\left(\bar{\Psi} \bar{\Omega}_{a b}\right) \bar{R}_{a b}+\frac{\log \Lambda}{3 \pi} \int\left(\bar{\Psi} \bar{\Omega}_{a b}\right) \bar{R}_{a c} G_{\text {fin }}^{b c} \\
& +\frac{\log \Lambda}{6 \pi} \int\left(\bar{\Psi} \bar{\Omega}_{a b}\right) G_{\text {fin }}^{c d}\left(\bar{\Omega}_{a c}^{\mu} \bar{\Omega}_{b d}^{\mu}-\bar{\Omega}_{a b}^{\mu} \bar{\Omega}_{c d}^{\mu}\right)+O\left(\alpha^{\prime}\right)
\end{aligned}
$$

In these formulas $\bar{R}_{a b}(\sigma)$ is the Ricci tensor of the induced metric, written in normal coordinates at the point $\bar{x}(\sigma)$. Some further details about the derivation of these formulas can be found in Appendix C.

The sum of 4.17)-(4.19) is equal to

$$
-\frac{\log \Lambda}{4 \pi^{2}} \int d \sigma\left(\bar{\Psi} \bar{\Omega}_{a b}\right) \bar{R}_{a b}+O\left(\alpha^{\prime}\right)
$$

In particular, just as we expected, the individual contributions of the unknown finite parts $G_{\text {fin }}^{a b}$ cancel in this sum.

\subsection{Corrected equations of motion}

Let me repeat the logic of the above computation. First I noticed that (4.2) contains a 1-loop divergence (4.12) of order $\alpha^{\prime 0}$. For this divergence to vanish modulo $\alpha^{\prime}$, I had to impose equation of motion (4.13). Then I proceeded to compute order $\alpha^{\prime}$ divergences coming from the 2-loop terms. In this computation I actually used (4.12) to show that many potentially divergent terms are of order $\alpha^{\prime 2}$ or higher and can be ignored. The result is that the total $\alpha^{\prime}$ divergence is equal to $4 \pi \alpha^{\prime}$ times (4.20). The divergent part of the $1 \mathrm{PI}$ effective action is

$$
F_{1 \mathrm{PI}}^{\mathrm{div}}=(4.12)+4 \pi \alpha^{\prime}(4.20)=\frac{\log \Lambda}{2 \pi} \int d \sigma \bar{\Psi}^{\mu}\left(\bar{\Omega}_{a a}^{\mu}-2 \alpha^{\prime} \bar{\Omega}_{a b}^{\mu} \bar{R}_{a b}\right)+O\left(\alpha^{\prime 2}\right) .
$$


Requiring that $F^{\text {div }}=O\left(\alpha^{\prime 2}\right)$, I get the corrected equation of motion

$$
\bar{\Omega}_{a a}^{\mu}-2 \alpha^{\prime} \bar{\Omega}_{a b}^{\mu} \bar{R}_{a b}=O\left(\alpha^{\prime 2}\right) .
$$

It is a simple matter to check that this equation of motion corresponds to the low energy effective action of the form

$$
S_{\mathrm{eff}}=T_{p} \int d^{p+1} x\left(\sqrt{G}+\alpha^{\prime} R \sqrt{G}\right)+O\left(\alpha^{\prime 2}\right) .
$$

Thus our computation predicts that there is an order $\alpha^{\prime}$ Einstein terms in the bosonic $\mathrm{D} p$-brane effective action, and fixes a relative coefficient in front of itt?

It remains to check that this coefficient agrees with the direct $S$-matrix analysis, as it should if our interpretation is correct.

\subsection{Dp-brane effective action from the S-matrix}

The open string vertex operators

$$
V^{i}(q)=\int d \sigma \partial_{\perp} X^{i} e^{i q_{a} X^{a}} \quad(a=0 \ldots p, i=p+1 \ldots 25)
$$

describe ripples on the $\mathrm{D} p$-brane polarized in the $i$-th transverse direction and propagating along the brane with momentum $q$. The 4-scattering amplitude for ripples with polarizations $i, j, k, l$ can be easily computed and is equal to

$$
\begin{gathered}
\mathcal{A}=\delta_{i j} \delta_{k l} A(s, t, u)+\delta_{i k} \delta_{j l} A(u, t, s)+\delta_{i l} \delta_{j k} A(t, s, u), \\
A(s, t, u)=B\left(-1-\alpha^{\prime} s, 1-\alpha^{\prime} t\right)+B\left(-1-\alpha^{\prime} s, 1-\alpha^{\prime} u\right)+B\left(1-\alpha^{\prime} u, 1-\alpha^{\prime} t\right) .
\end{gathered}
$$

Expanding in the region of small momenta and keeping the first $\alpha^{\prime}$ correction,

$$
\mathcal{A} \propto \delta_{i j} \delta_{k l}\left(t u-\alpha^{\prime} s t u\right)+\ldots+O\left(\alpha^{\prime 2}\right),
$$

where ... denotes the terms proportional to the other pairings.

\footnotetext{
${ }^{7}$ Notice that the Einstein term represents the only possible correction in this order. Dimensional analysis would also allow for $O\left(\alpha^{\prime}\right)$ terms containing the second fundamental form $\Omega_{a b}^{\mu}$ squared. However, the term $\left(\Omega_{a}^{\mu a}\right)^{2}$ is irrelevant on shell, since $\Omega_{a}^{\mu a}=0$ by the minimal surface equations of motion. The term $\Omega_{a b}^{\mu} \Omega^{\mu a b}$ is expressible via $R$ by the Gauss-Codazzi equations.

${ }^{8}$ After this paper was completed, it was brought to my attention by Arkady Tseytlin that the EinsteinHilbert term in the bosonic D-brane action was previously discovered in [28] by analyzing graviton scattering amplitudes in the presence of the brane. The relative coefficient in our formula (4.23) agrees with Eq. (3.16) in that paper.
} 
Eq. (4.26) has to be compared with the 4-ripple amplitude following from (4.23). To do the comparison, I must expand $S_{\text {eff }}$ to the second order in $g_{a b}=\partial_{a} \phi^{i} \partial_{b} \phi^{i}$. The actual computation is easy to perform using tetrads. The result is

$$
\begin{aligned}
S_{\text {eff }} \propto & \int 1+\frac{1}{2} g_{a a}+\frac{1}{8}\left(g_{a a}\right)^{2}-\frac{1}{4} g_{a b} g_{a b} \\
& +\alpha^{\prime}\left(\frac{1}{2} g_{a a} \partial_{b} \partial_{c} g_{b c}+\frac{1}{2} \partial_{a} g_{a b} \partial_{c} g_{c b}+\frac{1}{4} g_{a b} \partial^{2} g_{a b}-\frac{1}{4} g_{a a} \partial^{2} g_{b b}\right)+\ldots
\end{aligned}
$$

The indices are contracted with $\eta_{a b}$ in this formula.

As the reader may check, the 4-amplitude derived from (4.27) coincides with (4.26) exactly.

\section{Conclusion}

In this paper we demonstrated that reparametrization path-integrals are ubiquitous in string theory problems involving fixed space-time objects where the strings end, such as D-branes in critical string theory, or Wilson loops in gauge/string duality. For the first time we explored quantum properties of these integrals, laying out the foundations of any future treatment.

In some cases, such as for the Wilson loop on the boundary of AdS, these integrals give a finite contribution to the amplitude. In the others, such as for the D-branes, the integrals are UV divergent. However, in the latter case the integrals carry dynamical information related to the fact that the divergences can be removed by renormalizing the shape of the brane. This renormalization group flow is governed by the D-brane low energy effective action.

Many issues related to the above facts were investigated or checked only partially. Some of the more important open problems are related to the loop equations (see Section 2.4) and nonperturbative effects (see Section 3.1). Much further work and insight will be required to complete the emerging picture.

\section{Acknowledgements}

I would like to express deep gratitude to my advisor Alexander Polyakov for the ideas, knowledge, and wisdom he shared with me so generously and patiently. He introduced me to the subject, suggested the questions to study, and helped me interpret the results. This paper would have never been written without his constant support and attention. 
A part of this work was done while I was attending "Les Houches 2001". I am grateful to the organizers of the school and other participants for creating a wonderful scientific atmosphere.

I would like to thank Arkady Tseytlin for important comments about the first version of this paper. I would also like to thank Igor Klebanov and Juan Maldacena for the interesting and useful discussions of gauge/string duality in Les Houches and afterwards.

\section{Appendix A. Derivation of $(2.20)$}

Here we present a careful derivation of formula $(\overline{2.20})$ from the first principles. To begin with, let us introduce a boundary condition for the tangential component of the metric in (2.15)

$$
g_{\sigma \sigma}(\sigma, 0)=h(\sigma) .
$$

Thus $Z$ becomes a function of $c^{\mu}$ and $h$ (although conformal invariance would mean that the dependence on $h$ is actually not there.) With these definitions one can think of $Z$ as a sort of object obtained by cutting a closed string world-sheet into two halves:

$$
\left(\begin{array}{l}
0 \\
\vdots \\
1
\end{array}\right) \rightarrow\left(\begin{array}{c}
\vdots \\
\vdots \\
i
\end{array}\right)
$$

In particular, the closed string partition function formally factorizes

$$
\left(\begin{array}{l}
\vdots \\
\vdots
\end{array}=\int\left[\mathcal{D} c^{\mu} \mathcal{D} h\right]\left(Z\left[c^{\mu}, h\right]\right)^{2}\right.
$$

We proceed, as usual, to parametrize the metric $g$ in (2.15) by

$$
g_{a b}=\left(e^{\phi} \delta_{a b}\right)^{f}
$$

where $f$ is an upper half-plane diffeomorphism bringing $g$ to the conformal form. Because of (A.1), the pair $(\phi, f)$ is constrained by the boundary relation

$$
e^{\phi(\beta(\sigma))}\left[\beta^{\prime}(\sigma)\right]^{2}=h(\sigma),
$$

where $\beta(\sigma)=f^{1}(\sigma, 0)$. 
Now we change variables

$$
X^{\mu}(\xi)=\widetilde{X}^{\mu}(f(\xi))
$$

in the path-integral. The new field $\widetilde{X}$ satisfies the boundary condition

$$
\widetilde{X}^{\mu}(\sigma, 0)=c^{\mu}\left(\beta^{-1}(\sigma)\right)
$$

and has the action $\int(\partial \widetilde{X})^{2}$. So the path-integral over $\mathcal{D} \widetilde{X}$ is equal to

$$
\exp \left(-S_{\mathrm{cl}}\left[c^{\mu}\left(\beta^{-1}(\sigma)\right)\right]\right),
$$

where $S_{\mathrm{cl}}$ is given by (2.19). (We ignore conformal anomaly, which is going to cancel for $D=26$.) Thus, we get an intermediate result

$$
Z=\int[\mathcal{D} \phi \mathcal{D} f] \exp \left(-S_{\mathrm{cl}}\left[c^{\mu}\left(\beta^{-1}(\sigma)\right)\right]\right) .
$$

Now we would like to split $\mathcal{D} f$ into $\mathcal{D} \beta \mathcal{D} f_{0}$, where $f_{0} \in D i f f_{0}$ fixes the boundary. This splitting is local and does not introduce any Jacobian factor. The covariant measure of integration over $\mathcal{D} \beta$ is

$$
\|\delta \beta\|^{2}=\int d \sigma e^{3 \phi(\sigma) / 2}\left[\delta \beta\left(\beta^{-1}(\sigma)\right)\right]^{2} .
$$

Finally, let us make change of variables $\beta \rightarrow \alpha=\beta^{-1}$ in (A.9) and drop the infinite term $\int\left[\mathcal{D} \phi \mathcal{D} f_{0}\right]$. This gives us exactly (2.20).

The only thing that remains to figure out is the measure of integration in (2.20). Using

$$
\delta \beta\left(\beta^{-1}(\sigma)\right)=-\beta^{\prime}\left(\beta^{-1}(\sigma)\right) \delta \alpha(\sigma)
$$

and (A.5), it is easy to show that in terms of $\delta \alpha$ the measure (A.10) takes the form

$$
\|\delta \alpha\|^{2}=\int d \sigma h^{3 / 2}(\sigma)\left[\delta \alpha\left(\alpha^{-1}(\sigma)\right)\right]^{2} .
$$

This is the natural covariant measure of integration with fixed boundary metric $h(\sigma)$. This assures in particular that $Z$ is invariant with respect to simultaneous reparametrizations of $c^{\mu}$ and $h$ :

$$
Z\left[c^{\mu}, h\right]=Z\left[c^{\mu}(\alpha(\sigma)), h(\alpha(\sigma))\left(\alpha^{\prime}(\sigma)\right)^{2}\right] .
$$


In the main text of the paper we chose to suppress the implicit dependence of $Z$ on $h$ contained in the measure, and detected conformal anomalies by looking at the logarithmic divergences. An alternative way would be to analyze the dependence of the finite part of (2.20) on $h(\sigma)$. This dependence comes out to be nonzero and proportional to

$$
\int d \sigma \frac{\delta S_{\mathrm{cl}}}{\delta c^{\mu}(\sigma)} \frac{\ddot{c}_{\mu}}{\dot{c}^{2}} \log h(\sigma)
$$

in the 1-loop approximation. This leads to the same conclusion that (2.15) is not conformally invariant.

\section{Appendix B. D0-brane divergences}

In this appendix we derive formulas (3.58) and (3.59) and explain why the diagrams in (3.52) are convergent.

The l.h.s. of (3.58) is equal to

$$
\frac{\log \Lambda}{2 \pi} \iint K_{\sigma \sigma^{\prime}} G\left(\sigma, \sigma^{\prime}\right) c_{\mu}^{\prime}(\sigma) c_{\mu}^{\prime}\left(c^{\prime} c^{\prime \prime \prime}\right)\left(\sigma^{\prime}\right)+\iint K_{\sigma \sigma^{\prime}} G\left(\sigma, \sigma^{\prime}\right) c_{\mu}^{\prime}(\sigma) c_{\mu}^{\prime \prime \prime} G_{\text {fin }}\left(\sigma^{\prime}\right)
$$

The only divergence of the second term comes from the oyster diagram (3.56), (3.57). This gives us half the second term in the r.h.s. of (3.58). The first term in (B.1) contains both $(\log \Lambda)^{2}$ and $\log \Lambda$ divergences. It can in fact be evaluated exactly using the fact that $G\left(\sigma, \sigma^{\prime}\right)$ satisfies (3.43). Indeed, putting $\sigma=\sigma^{\prime \prime}$ in (3.43) gives

$$
c_{\mu}^{\prime}(\sigma) \int d \sigma^{\prime} K_{\sigma \sigma^{\prime}} c_{\mu}^{\prime}\left(\sigma^{\prime}\right) G\left(\sigma^{\prime}, \sigma\right)+\left(\psi c^{\prime \prime}\right)(\sigma) G(\sigma, \sigma)=\frac{1}{2} \delta(0)
$$

All three terms in this equation are divergent. However, the divergence in $\delta(0)=\Lambda / 2 \pi$ is purely linear and can be discarded. Now we can use (B.2) to conclude that in the regulated theory

$$
c_{\mu}^{\prime}(\sigma) \int d \sigma^{\prime} K_{\sigma \sigma^{\prime}} c_{\mu}^{\prime}\left(\sigma^{\prime}\right) G\left(\sigma^{\prime}, \sigma\right)=-\left(\frac{\log \Lambda}{2 \pi}+G_{\mathrm{fin}}(\sigma)\right)\left(\psi c^{\prime \prime}\right)(\sigma)
$$

Substituting this into (B.1) gives us the first term and the second half of the second term in the r.h.s. of (3.58).

Now let us turn to (3.59). This diagram is most conveniently analyzed in momentum space. Since the Green's function $G(\sigma, p)$ depends on $\sigma$, the usual Feynman rules have 
to be modified. A second momentum $v$ (dual to $\sigma$ ) is associated with every propagator, and $+v / 2$ contributes to momentum conservation in both vertices joined by it. These properties follow from the representation

$$
G\left(\sigma, \sigma^{\prime}\right)=\iint \frac{d v d p}{(2 \pi)^{2}} G(v, p) e^{i v \frac{\sigma+\sigma^{\prime}}{2}+i p\left(\sigma-\sigma^{\prime}\right)}
$$

The momentum flow in our particular case is

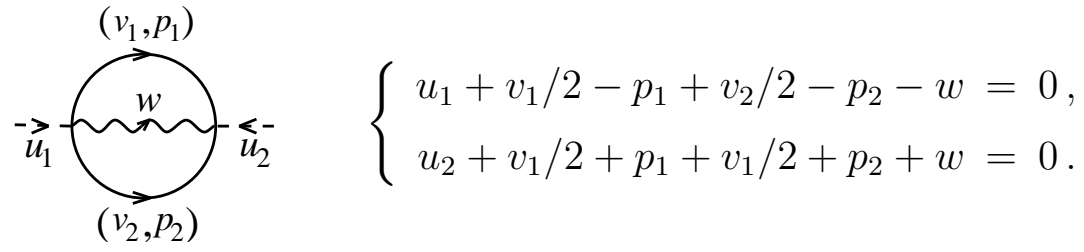

Excluding $w$, we get the following expression for the diagram

$$
\begin{aligned}
\cdots- & =\frac{1}{4} \int \frac{d^{2} u d^{2} v}{(2 \pi)^{4}} 2 \pi \delta\left(u_{1}+u_{2}+v_{1}+v_{2}\right) c_{\mu}^{\prime \prime}\left(u_{1}\right) c_{\mu}^{\prime \prime}\left(u_{2}\right) J\left(u_{1}, u_{2}, v_{1}, v_{2}\right), \\
J & =\int \frac{d^{2} p}{(2 \pi)^{2}}\left|p_{1}+p_{2}-u_{1}-\frac{v_{1}+v_{2}}{2}\right| G\left(v_{1}, p_{1}\right) G\left(v_{2}, p_{2}\right) .
\end{aligned}
$$

I regulate the divergent integral $J$ by imposing the cut-off $\left|p_{i}\right|<\Lambda$. Using (3.55) it is easy to see that

$$
J=\int \frac{d^{2} p}{(2 \pi)^{2}}\left|p_{1}+p_{2}\right| G\left(v_{1}, p_{1}\right) G\left(v_{2}, p_{2}\right)+\text { finite }
$$

so that the divergent part of $J$ is actually independent of $u_{1}$ and $u_{2}$.

Let us split the region of integration into $\left|p_{2}\right|<\left|p_{1}\right|$ and $\left|p_{2}\right|>\left|p_{1}\right|$. In the first case

$$
\left|p_{1}+p_{2}\right|=\left|p_{1}\right|+p_{2} \operatorname{sign} p_{1}
$$

It can be shown using (3.55) that the second term leads to a convergent integral in (B.7), so that

$$
J_{\left|p_{2}\right|<\left|p_{1}\right|}=\int_{\left|p_{1}\right|<\Lambda} \frac{d p_{1}}{2 \pi}\left|p_{1}\right| G\left(v_{1}, p_{1}\right) \int_{\left|p_{2}\right|<\left|p_{1}\right|} \frac{d p_{2}}{2 \pi} G\left(v_{2}, p_{2}\right)+\text { finite. }
$$

Expansion of the inner integral for $\left|p_{1}\right| \rightarrow \infty$ is easy to obtain from (3.55)

$$
\int_{\left|p_{2}\right|<\left|p_{1}\right|} \frac{d p_{2}}{2 \pi} G\left(v_{2}, p_{2}\right)=\delta\left(v_{2}\right) \ln \left|p_{1}\right|+G_{\text {fin }}\left(v_{2}\right)+\frac{\left(\psi c^{\prime \prime}\right)\left(v_{2}\right)}{2 \pi\left|p_{1}\right|}+\ldots
$$


The $O\left(p_{1}^{-2}\right)$ error term will not contribute to the divergence. By (3.55) and (B.10) we know the asymptotics of the integrand in $(\mathbb{B . 9})$. The divergence of $J$ follows from this information in a straightforward way

$$
\begin{aligned}
J_{\left|p_{2}\right|<\left|p_{1}\right|}= & -\frac{(\log \Lambda)^{2}}{8 \pi^{2}}\left(\psi c^{\prime \prime}\right)\left(v_{1}\right) 2 \pi \delta\left(v_{2}\right) \\
& +\frac{\log \Lambda}{4 \pi^{2}} 2 \pi \delta\left(v_{1}\right)\left(\psi c^{\prime \prime}\right)\left(v_{2}\right)-\frac{\log \Lambda}{2 \pi}\left(\psi c^{\prime \prime}\right)\left(v_{1}\right) G_{\text {fin }}\left(v_{2}\right)+\text { finite },
\end{aligned}
$$

where I also omitted divergent terms proportional to $\Lambda$ and $\Lambda \log \Lambda$. The $\left|p_{2}\right|>\left|p_{1}\right|$ part of $J$ is obtained from the $\left|p_{2}\right|<\left|p_{1}\right|$ part by simply interchanging $v_{1} \leftrightarrow v_{2}$.

Substituting (B.11) into (B.6), we get the divergence of the diagram. For example, the $(\log \Lambda)^{2}$ term comes out to be

$$
-\frac{(\log \Lambda)^{2}}{16 \pi^{2}} \int\left(\psi c^{\prime \prime}\right) c^{\prime \prime 2}
$$

Analogously all the other terms reduce to single $d \sigma$ integrals, and we arrive at (3.59).

Finally, let us show that the diagrams in (3.52) are convergent. For the last diagram it is true simply because $G^{a b}(\sigma, p) \sim|p|^{-1}$. Since $K(p)=|p|$, the other three diagrams in (3.52) could in principle diverge. However, notice that the kernel $K_{\sigma \sigma^{\prime}}$ appears in all of them in the combination

$$
A\left(\sigma, \sigma^{\prime}\right)=c_{\mu}^{\prime}(\sigma) c_{\mu}^{\prime \prime}\left(\sigma^{\prime}\right) K_{\sigma \sigma^{\prime}}
$$

The coefficient of the leading $p \rightarrow \infty$ singularity of this expression

$$
A(\sigma, p)=\left(c^{\prime} c^{\prime \prime}\right)(\sigma)|p|+\ldots
$$

vanishes identically. So in fact

$$
A(\sigma, p) \sim \operatorname{sign} p+\ldots \quad(p \rightarrow \infty)
$$

from which it follows that the remaining three diagrams in (3.52) are also finite.

\section{Appendix C. D $p$-brane divergences}

$\mathrm{D} p$-brane divergences are analyzed analogously to the D0-brane case. In this appendix we will indicate minor differences in the analysis and record Riemannian geometry identities needed to bring the answers to the form (4.17)-(4.19). 
Let us start with the diagram

$$
\begin{aligned}
- & =\frac{1}{12} \int d \sigma\left(\bar{\Psi} \bar{M}_{a b c d}\right) G^{a b}(\sigma, \sigma) G^{c d}(\sigma, \sigma) \\
& =\frac{(\log \Lambda)^{2}}{48 \pi^{2}} \int\left(\bar{\Psi} \bar{M}_{a a b b}\right)+\frac{\log \Lambda}{12 \pi} \int\left(\bar{\Psi} \bar{M}_{a a b c}\right) G_{\text {fin }}^{b c}+\text { finite }
\end{aligned}
$$

where I used (4.11) to find the divergent part.

It is not difficult to find by commuting covariant derivatives that?

$$
\begin{aligned}
& \bar{M}_{a a b b}^{\mu}=\frac{2}{3} \bar{\Omega}_{a b}^{\mu} \bar{R}_{a b}+\ldots \\
& \bar{M}_{a a b c}^{\mu}=\frac{2}{3}\left(\bar{\Omega}_{a d}^{\mu} \bar{R}_{a b c d}+\bar{\Omega}_{a b}^{\mu} \bar{R}_{a c}+\bar{\Omega}_{a c}^{\mu} \bar{R}_{a b}\right)+\ldots
\end{aligned}
$$

where the omitted terms that are either $O\left(\alpha^{\prime}\right)$ by the use of (4.13), or proportional to $\bar{E}^{\mu}$. The latter terms are irrelevant in (C.1) because of (4.9).

Now if we express the Riemann tensor by the Gauss-Codazzi equation

$$
\bar{R}_{a b c d}=\bar{\Omega}_{a c}^{\nu} \bar{\Omega}_{b d}^{\nu}-\bar{\Omega}_{a d}^{\nu} \bar{\Omega}_{b c}^{\nu}
$$

we get exactly Eq. (4.19).

Let us turn to

$$
\begin{aligned}
\text {. }- & \frac{\log \Lambda}{6 \pi} \iint K_{\sigma \sigma^{\prime}} G^{a b}\left(\sigma, \sigma^{\prime}\right) \bar{E}_{a}^{\mu}(\sigma) \bar{L}_{b c c}^{\mu}\left(\sigma^{\prime}\right) \\
& +\frac{1}{3} \iint K_{\sigma \sigma^{\prime}} G^{a b}\left(\sigma, \sigma^{\prime}\right) \bar{E}_{a}^{\mu}(\sigma) \bar{L}_{b c d}^{\mu}\left(\sigma^{\prime}\right) G_{\text {fin }}^{c d}\left(\sigma^{\prime}\right) .
\end{aligned}
$$

The divergence in the second term is proportional to the oyster diagram

$$
\oiiint=\int \frac{d p}{2 \pi}|p| G^{a b}(\sigma, p)=-\frac{1}{2 \pi} \log \Lambda\left(\bar{\Psi} \bar{\Omega}_{a b}\right)(\sigma)+\text { finite }
$$

and is equal to

$$
-\frac{\log \Lambda}{6 \pi} \int\left(\bar{\Psi} \bar{\Omega}_{a b}\right) \bar{E}_{a}^{\mu} \bar{L}_{b c d}^{\mu} G_{\text {fin }}^{c d}
$$

The coefficient $\bar{L}_{b c d}^{\mu}$ can be excluded using the easily derived identity

$$
\bar{E}_{a}^{\mu} \bar{L}_{b c d}^{\mu}=-\frac{1}{3}\left(\bar{\Omega}_{a b}^{\mu} \bar{\Omega}_{c d}^{\mu}+\bar{\Omega}_{a c}^{\mu} \bar{\Omega}_{b d}^{\mu}+\bar{\Omega}_{a d}^{\mu} \bar{\Omega}_{b c}^{\mu}\right) .
$$

\footnotetext{
${ }^{9}$ The Riemann tensor sign convention is $\left(\nabla_{b} \nabla_{a}-\nabla_{a} \nabla_{b}\right) A_{d}=A_{c} R_{d[a b]}^{c}$.
} 
The same argument applied to the first term in (C.4) gives me only the $(\log \Lambda)^{2}$ part of its divergence

$$
-\frac{(\log \Lambda)^{2}}{12 \pi} \int\left(\bar{\Psi} \bar{\Omega}_{a b}\right) \bar{E}_{a}^{\mu} \bar{L}_{b c c}^{\mu} .
$$

To find the subleading $\log \Lambda$ part, a more refined analysis is required. Commuting derivatives gives

$$
\bar{L}_{b c c}^{\mu}=\frac{2}{3} \bar{E}_{c}^{\mu} \bar{R}_{b c}+O\left(\alpha^{\prime}\right) .
$$

This suggests to invoke the Green's function defining equation

$$
\bar{E}_{a}^{\mu}(\sigma) \int d \sigma^{\prime} K_{\sigma \sigma^{\prime}} \bar{E}_{b}^{\mu}\left(\sigma^{\prime}\right) G^{b c}\left(\sigma^{\prime}, \sigma^{\prime \prime}\right)+\left(\bar{\Psi} \bar{\Omega}_{a b}\right)(\sigma) G^{b c}\left(\sigma, \sigma^{\prime \prime}\right)=\frac{1}{2} \delta\left(\sigma-\sigma^{\prime \prime}\right) \delta_{a c} .
$$

to evaluate the first integral in (C.4). Putting $\sigma=\sigma^{\prime \prime}$ and ignoring the purely linear divergence coming from $\delta(0)$ gives

$$
\bar{E}_{a}^{\mu}(\sigma) \int d \sigma^{\prime} K_{\sigma \sigma^{\prime}} \bar{E}_{b}^{\mu}\left(\sigma^{\prime}\right) G^{b c}\left(\sigma^{\prime}, \sigma\right)=-\frac{\log \Lambda}{2 \pi}\left(\bar{\Psi} \bar{\Omega}_{a c}\right)(\sigma)-\left(\bar{\Psi} \bar{\Omega}_{a b}\right) G_{\text {fin }}^{b c}(\sigma) .
$$

Using (C.11) and (C.9), I can find the complete logarithmic divergence of the first term in (C.4). It is equal to

$$
-\frac{(\log \Lambda)^{2}}{18 \pi} \int\left(\bar{\Psi} \bar{\Omega}_{a b}\right) \bar{R}_{a b}-\frac{\log \Lambda}{9 \pi} \int\left(\bar{\Psi} \bar{\Omega}_{a b}\right) \bar{R}_{a c} G_{\text {fin }}^{b c}+O\left(\alpha^{\prime}\right) .
$$

The total divergence of the diagram is equal to the sum of (C.6) and (C.12), and coincides with the answer given in Eq. (4.18).

The remaining diagram (4.17) is analyzed identically to the D0-brane case. One has to use the relation

$$
\bar{R}_{a b}=-\bar{\Omega}_{a c}^{\mu} \bar{\Omega}_{b c}^{\mu}+O\left(\alpha^{\prime}\right),
$$

which follows from (C.3) and (4.13), to transform the answer to the form given in (4.17). I omit the details.

\section{References}

[1] K. G. Wilson, "Confinement of quarks," Phys. Rev. D10 (1974) 2445-2459

[2] A. M. Polyakov, "Gauge fields as rings of glue," Nucl. Phys. B164 (1980) 171-188 
[3] A. M. Polyakov, "String theory and quark confinement," Nucl. Phys. Proc. Suppl. 68 (1998) 1-8, hep-th/9711002

[4] A. M. Polyakov, "The wall of the cave," Int. J. Mod. Phys. A14 (1999) 645-658, hep-th/9809057

[5] A. M. Polyakov, "Gauge Fields and Strings," Harwood Academic Publishers (1987)

[6] A. Cohen, G. Moore, P. Nelson, J. Polchinski, "An off-shell propagator for string theory," Nucl. Phys. B267 (1986) 143-157

[7] J. Maldacena, "Wilson loops in large $N$ field theories," Phys. Rev. Lett. 80 (1998) 4859-4862, hep-th/9803002

[8] S.-J. Rey, J. Yee, "Macroscopic strings as heavy quarks in large $N$ gauge theory and anti-de Sitter supergravity," Eur. Phys. J. C22 (2001) 379-394, hep-th/9803001

[9] S. S. Gubser, I. R. Klebanov, A. M. Polyakov, "Gauge theory correlators from noncritical string theory," Phys. Lett. B428 (1998) 105-114, hep-th/9802109

[10] A. M. Polyakov, "String theory as a universal language," Phys. Atom. Nucl. 64 (2001) 540-547, hep-th/0006132

[11] O. Aharony, S. S. Gubser, J. Maldacena, H. Ooguri, Y. Oz, "Large $N$ field theories, string theory and gravity," Phys. Rept. 323 (2000) 183-386, hep-th/9905111

[12] I. R. Klebanov, M. J. Strassler, "Supergravity and a confining gauge theory: Duality cascades and $\chi$ SB-resolution of naked singularities," JHEP 08 (2000) 052, hep-th/ 0007191

[13] A. M. Polyakov, "Gauge fields and space-time," hep-th/0110196

[14] Yu. M. Makeenko, A. A. Migdal, "Exact equation for the loop average in multicolor QCD," Phys. Lett. B88 (1979) 135, [Erratum - ibid. B89 (1980) 437]

[15] A. A. Migdal, "Loop equations and 1/N expansion," Phys. Rept. 102 (1983) 199-290

[16] V. S. Dotsenko, S. N. Vergeles, "Renormalizability of phase factors in the nonabelian gauge theory," Nucl. Phys. B169 (1980) 527-546

[17] R. A. Brandt, A. Gocksch, M. A. Sato, F. Neri, "Loop space," Phys. Rev. D26 (1982) $3611-3640$

[18] A. M. Polyakov, V. S. Rychkov, "Gauge fields-strings duality and the loop equation," Nucl. Phys. B581 (2000) 116-134, hep-th/0002106 
[19] A. M. Polyakov, V. S. Rychkov, "Loop dynamics and AdS/CFT correspondence," Nucl. Phys. B594 (2001) 272-286, hep-th/0005173

[20] J. K. Erickson, G. W. Semenoff, K. Zarembo, "Wilson loops in $\mathcal{N}=4$ supersymmetric Yang-Mills theory," Nucl. Phys. B582 (2000) 155-175, hep-th/0003055

[21] N. Drukker, D. J. Gross, "An exact prediction of $\mathcal{N}=4$ SUSYM theory for string theory," J. Math. Phys. 42 (2001) 2896-2914, hep-th/0010274

[22] N. Drukker, D. J. Gross, A. A. Tseytlin, "Green-Schwarz string in $A d S_{5} \times S^{5}$ : Semiclassical partition function," JHEP 04 (2000) 021, hep-th/0001204

[23] S. Forste, D. Ghoshal, S. Theisen, "Stringy corrections to the Wilson loop in $\mathcal{N}=4$ super Yang-Mills theory," JHEP 08 (1999) 013, hep-th/9903042

[24] C. G. Callan, E. J. Martinec, M. J. Perry, D. Friedan, "Strings in background fields," Nucl. Phys. B262 (1985) 593-609

[25] R. G. Leigh, "Dirac-Born-Infeld action from Dirichlet $\sigma$-model," Mod. Phys. Lett. A4 (1989) 2767-2772

[26] T. S. Bunch, L. Parker, "Feynman propagator in curved space-time: A momentum space representation," Phys. Rev. D20 (1979) 2499-2510

[27] D. H. Friedan, "Nonlinear models in $2+\varepsilon$ dimensions," Ann. Phys. 163 (1985) $318-419$

[28] S. Corley, D. A. Lowe, S. Ramgoolam, "Einstein-Hilbert action on the brane for the bulk graviton," JHEP 07 (2001) 030, hep-th/0106067 